\title{
The UDP-GalNAcA biosynthesis genes gna-gne2 are required to maintain cell envelope integrity and in vivo fitness in multi-drug resistant Acinetobacter baumannii
}

\author{
Sébastien Crépin, ${ }^{1}$ Elizabeth N. Ottosen, ${ }^{1}$ \\ Courtney E. Chandler, ${ }^{2}$ Anna Sintsova, ${ }^{1}$ \\ Robert K. Ernst ${ }^{2}$ and Harry L. T. Mobley (iD) ${ }^{1 *}$ \\ ${ }^{1}$ Department of Microbiology and Immunology, University \\ of Michigan Medical School, Ann Arbor, MI, USA. \\ ${ }^{2}$ Department of Microbial Pathogenesis, School of \\ Dentistry, University of Maryland, Baltimore, MD, USA.
}

\section{Summary}

Acinetobacter baumannii infects a wide range of anatomic sites including the respiratory tract and bloodstream. Despite its clinical importance, little is known about the molecular basis of $A$. baumannii pathogenesis. We previously identified the UDP$\mathrm{N}$-acetyl-D-galactosaminuronic acid (UDP-GalNAcA) biosynthesis genes, gna-gne2, as being critical for survival in vivo. Herein, we demonstrate that GnaGne2 are part of a complex network connecting in vivo fitness, cell envelope homeostasis and resistance to antibiotics. The $\Delta$ gna-gne 2 mutant exhibits a severe fitness defect during bloodstream infection. Capsule production is abolished in the mutant strain, which is concomitant with its inability to survive in human serum. In addition, the $\Delta$ gna-gne 2 mutant was more susceptible to vancomycin and unable to grow on MacConkey plates, indicating an alteration in cell envelope integrity. Analysis of lipid A by mass spectrometry showed that the hexa- and hepta-acylated species were affected in the gna-gne2 mutant. Finally, the $\Delta$ gna-gne2 mutant was more susceptible to several classes of antibiotics. Together, this study demonstrates the importance of UDP-GalNAcA in the pathobiology of $A$. baumannii. By interrupting its biosynthesis, we showed that this molecule plays a critical role in capsule biosynthesis and maintaining the cell envelope homeostasis.

*For correspondence. E-mail hmobley@umich.edu; Tel.1-734-7631058; Fax 1-734-763-7163.

\section{Introduction}

Acinetobacter baumannii, an encapsulated gram-negative bacterium, has emerged as a prominent and dangerous nosocomial pathogen (Munoz-Price and Weinstein, 2008; Wong et al., 2017). A. baumannii is a serious threat among immunocompromised individuals as well as patients in intensive and specialized post-operative care units (Dijkshoorn et al., 2007; Wong et al., 2017; Harding et al., 2018). This pathogen can cause a wide range of infections including those of the respiratory tract, bloodstream and wounds (Wong et al., 2017). Annually, an average of 62,200 and 1,000,000 infections are attributed to $A$. baumannii in the United States and worldwide, respectively (Spellberg and Rex, 2013). Additionally, the mortality rate associated with $A$. baumannii infections ranges from 36 to $50 \%$ (Seifert et al., 1995; Fagon et al., 1996; Garnacho et al., 2003; Wisplinghoff et al., 2004). The high prevalence of infection and increasing antibiotic resistance leave few, if any, treatment options and exacerbate the threat of this deadly pathogen. Accordingly, the World Health Organization (WHO) positioned A. baumannii as a top priority for which new antimicrobials are urgently needed (Lawe-Davies and Bennett, 2017; Willyard, 2017). However, despite its clinical importance, the mechanisms by which $A$. baumannii infect and survive in the host are poorly understood.

Until recently, just a subset of virulence factors had been identified, including capsule, lipooligosaccharide, metal acquisition systems (iron and zinc), secretion systems (Type I, II and VI), the phenotypic switch and a lytic transglycosylase (Wong et al., 2017; Chin et al., 2018; Crépin et al., 2018; Harding et al., 2018; Waack et al., 2018; Runci et al., 2019). However, the regulation of these factors and their specific roles in the pathobiology of $A$. baumannii infections are yet to be elucidated. Using a combination of transposon- and RNA-sequencing (Tn-seq and RNA-seq), multiple strain backgrounds and animal models of infection, we and others have identified the full set of candidate factors contributing to A. baumannii pathogenesis (Wang et al., 2014; Gebhardt et al., 2015; Subashchandrabose et al., 2016; Crépin et al., 2018). 
In strain $\mathrm{AB} 0057$, genes belonging to the $\mathrm{K}$ locus (Kenyon and Hall, 2013; Crépin et al., 2018), which comprises genes involved in capsule biosynthesis, were identified as candidate fitness factors (Kenyon and Hall, 2013; Crépin et al., 2018). The gna-gne2 genes (also referred as $t v i B C$ ), which are involved in the biosynthesis of UDP$\mathrm{N}$-acetyl-D-galactosaminuronic acid (UDP-GalNAcA), play a crucial role during bloodstream infection (Crépin et al., 2018). Additionally, UDP-GalNAcA is a component of the polysaccharide capsule in several other strains of $A$. baumannii, such as 307-0294, 28, RBH2, LUH5535 and D23 (Russo et al., 2013; Kenyon et al., 2016; Shashkov et al., 2017; 2018).

Surface carbohydrates, such as capsule, lipopolysaccharide (LPS)/lipooligosaccharide (LOS), and glycoproteins are known to play key roles in bacterial physiology and pathogenesis. Capsules ( $\mathrm{K}$ antigens) are surfaceexposed structures enveloping bacteria, and are composed of repeating oligosaccharide subunits ( $K$ units). Capsule composition is widely diverse with numerous structures already described. For example, around 80 capsule types have been identified in $E$. coli, while over 100 types have been described in A. baumannii (Whitfield, 2006; Singh et al., 2019). Capsules are well-established virulence factors, protecting the cells against harsh environments, promoting immune evasion and aiding in antimicrobial resistance (Whitfield, 2006; Tipton et al., 2018; Singh et al., 2019).

In Gram-negative bacteria, LPS is the main component of the outer membrane (OM). LPS is divided into three parts: a variable polysaccharide chain known as the O-antigen, a core oligosaccharide and lipid A (Raetz and Whitfield, 2002). The lipid A moiety anchors the LPS structure to the OM. LPS plays a critical role in protecting the cells against the host immune defenses and noxious compounds such as cationic antimicrobial peptides and antibiotics (Raetz and Whitfield, 2002; Simpson and Trent, 2019). Rather than LPS, A. baumannii synthesizes LOS, an analogous version of LPS lacking the O-antigen (Powers and Trent, 2018).

Synthesis of UDP-GalNAcA begins with the conversion of UDP-N-acetylglucosamine (UDP-GlcNAc) to UDP- $N$-acetylglucosaminuronic acid (UDP-GIcNAcA) by the UDP- $N$-acetylglucosamine C- 6 dehydrogenase Gna. UDP-GICNACA is then converted to UDP-GalNAcA by Gne2, a UDP-N-acetylglucosaminuronic acid C-4 epimerase (Zhang et al., 2006). A. baumannii AB0057 Gna has $75 \%$ amino acid sequence identity and $87 \%$ amino acid similarity with the Pseudomonas aeruginosa Gna (WpbO) homolog, while Gne2 from AB0057 has 73\% identity and $86 \%$ similarity with the $P$. aeruginosa Gne2 (WpbP) homolog (Table S1). Similar trends are observed with the corresponding homologs in Salmonella enterica serovar Typhi (Table S1).
In S. Typhi, UDP-GalNAcA is the monomeric precursor of the Vi capsular antigen (Johnson et al., 1965; Hashimoto et al., 1993; Waxin et al., 1993; Zhang et al., 2006), a crucial virulence factor and the target of all current vaccines against this pathogen. In $P$. aeruginosa, UDP-GalNAcA is a component of the O-antigen B-band (Knirel, 1990; Belanger et al., 1999; Lam et al., 2011). Interestingly, A. baumannii produces neither the Vi- or $\mathrm{O}$-antigens. As the biosynthesis of UDP-GalNAcA is important for colonization of the bloodstream in $A$. baumannii strain AB0057, (Crépin et al., 2018), this study aims to determine, using a genetic approach, the contribution of its biosynthesis genes to $A$. baumannii pathobiology. First, a double gna-gne2 mutant (referred here as mutant $\triangle a / e 2)$ was constructed to abolish biosynthesis of UDP-GalNAcA. Then, single gna and gne2 mutants were created to test the independent contribution of both genes to the tested phenotypes. Herein, we demonstrate that the UDP-GaINACA biosynthesis genes gna-gne2 are part of a complex network connecting capsule biosynthesis, cell envelope integrity, in vivo fitness and antibiotic resistance. We also determine that the phenotypes observed in the $\Delta a / e 2$ mutant were largely attributed to the loss of gna.

\section{Results}

The gna-gne2 tandem is conserved among A. baumannii bloodstream isolates

Previous Tn-seq and RNA-seq screens identified genes belonging to the $\mathrm{K}$ locus as candidate fitness factors (Kenyon and Hall, 2013) (Fig. 1A, Table S2 and [Wang et al., 2014; Gebhardt et al., 2015; Subashchandrabose et al., 2016; Murray et al., 2017; Crépin et al., 2018]). In strain AB0057 (KL4), gne2 (tviC) showed the greatest fitness defect in the spleen, -445.7 -fold and showed the 50th greatest fitness defect overall (Crépin et al., 2018). In this strain, gne2 is encoded in an operon with gna (tviB) (fitness defect of 119.4-fold in the spleen). Gna, a member of the dehydrogenase family, and Gne2, a member of the epimerase superfamily, are involved in the biosynthesis of UDP-GalNACA, which has been shown to be a component of the capsule in A. baumannii strains 3070294, 28, RBH2, LUH5535 and D23 (Russo et al., 2013; Kenyon et al., 2016; Shashkov et al., 2017; 2018).

The distribution of gna and gne2 across $A$. baumannii strains was evaluated. By removing the partial genomes, and the ones with atypical sizes and anomalous sequences, 2,991 genomes from the NCBI genome database were surveyed (https://www.ncbi.nlm.nih.gov/genom e/genomes/403. Accessed 25 October 2019). A gene was considered present if its corresponding homolog, from other strains, was $\geq 70 \%$ identical (protein sequence) with $\geq 90 \%$ coverage of the entire sequence. The analysis 


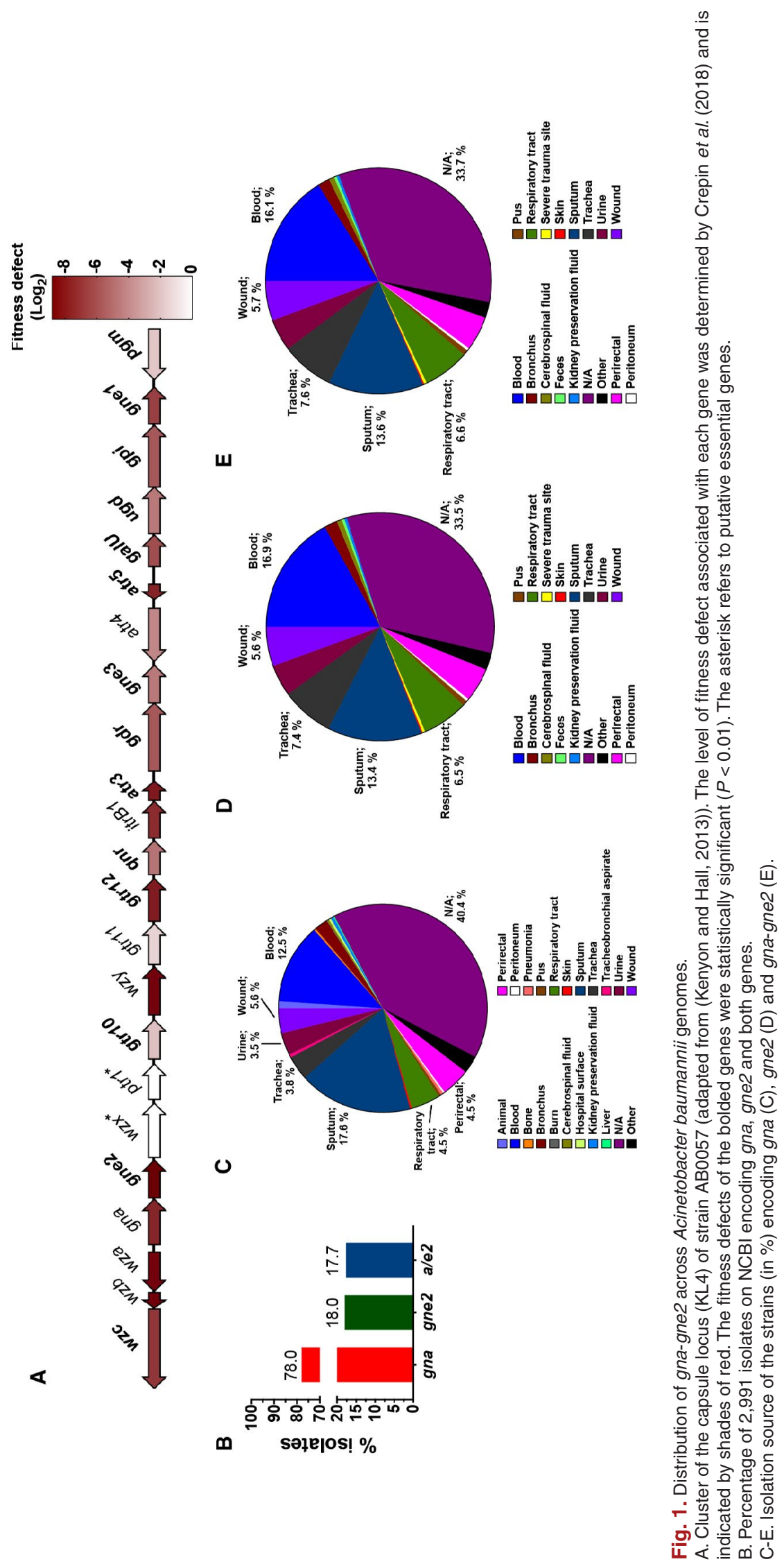

(C) 2019 John Wiley \& Sons Ltd, Molecular Microbiology, 113, 153-172 
revealed that gna is present in $78 \%$ of strains and is primarily associated with sputum (17.6\%) and blood (12.5\%) isolates (Fig. 1B-C). The analysis also showed that gne2 is present in $18 \%$ of strains and is mostly associated with blood (16.9\%) and sputum (13.4\%) isolates (Fig. 1B-D). Ninety-eight percent of strains encoding gne2 also encode gna, indicating that, when present, gne2 is predominantly associated with gna (Fig. 1B). Furthermore, the gna-gne2 tandem is primarily associated with blood (16.1\%) and sputum (13.6\%) isolates (Fig. 1E).

\section{Gna-Gne2 are crucial during bloodstream infection}

In strain AB0057, we previously demonstrated that interrupting UDP-GaINAcA biosynthesis dramatically decreased the ability of the mutant to colonize the murine bloodstream (Crépin et al., 2018). To confirm our initial screen, and that gna-gne2 are indeed essential during bloodstream infection, the double gna-gne2 mutant $(\Delta a / e 2)$ was complemented by cloning the gna-gne2 genes, including their native promoter, into the complementing vector pABBR_Km (Crépin et al., 2018), creating vector pABBR_Km-a/e2 and transforming into the a/e2 mutant strain. The in vivo fitness of the wild-type (WT), $\Delta a / e 2$ and the complemented strain was evaluated using the neutropenic murine model of bacteremia. Mice were inoculated with $10^{7} \mathrm{CFU}$ of each strain by tail-vein injection, and at $24 \mathrm{~h}$ post-inoculation, colonization of the spleen, liver and kidneys was determined by CFU enumeration. Compared to the WT strain, the $\Delta a / e 2$ mutant exhibited a fitness defect of 4.0-, 3.2- and 3.3-logs in the spleen, liver and kidneys, respectively (Fig. 2A). In trans complementation of the mutant restored fitness to WT levels in all organs (Fig. 2A), confirming that gna-gne2 are critical during bloodstream infection.

To determine the independent contribution of gna and gne2 to in vivo fitness, in-frame, markerless deletion mutants of gna and gne2 were then constructed and
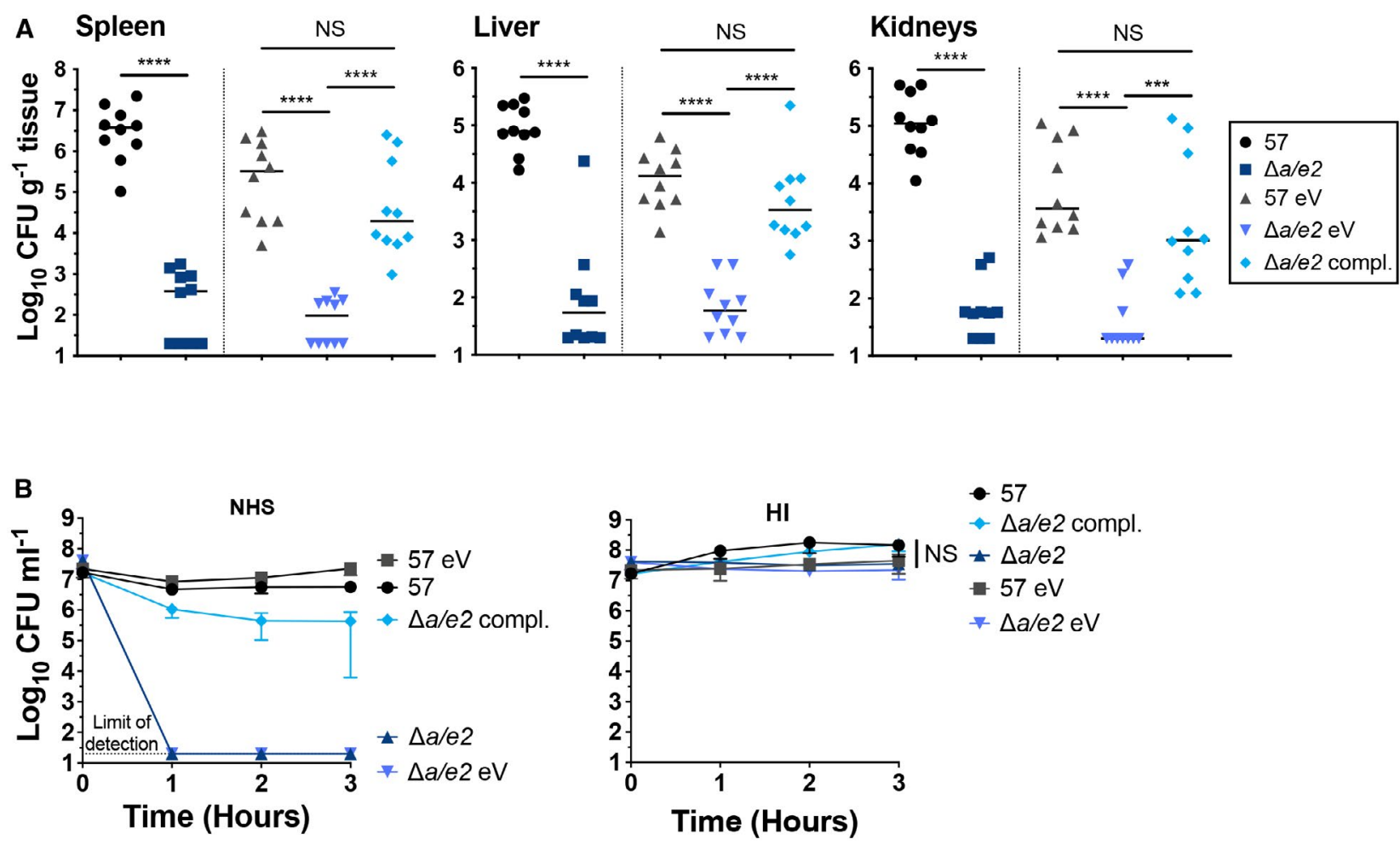

Fig. 2. Colonization of the bloodstream by the gna-gne2 (a/e2) mutant.

A. Colonization of the spleen, liver and kidneys was determined by inoculating CBA/J mice by tail vein injection with $10^{7} \mathrm{CFU}$ of either the WT strain (57) or its derivative strains. At $24 \mathrm{hpi}$, mice were sacrificed, organs were harvested, and the bacterial burden was determined by CFU enumeration on LB agar (57 and $\Delta \mathrm{a} / \mathrm{e} 2$ ) or LB-Km agar ( $57 \mathrm{eV}, 57 \Delta \mathrm{a} / \mathrm{e} 2 \mathrm{eV}$ and $57 \Delta \mathrm{a} / \mathrm{e} 2 \mathrm{compl}$.). Bacterial numbers are presented as the Log ${ }_{10}$ CFU $\mathrm{g}^{-1}$ of tissue. Each data point represents a sample from an individual mouse, and horizontal bars indicate the median values.

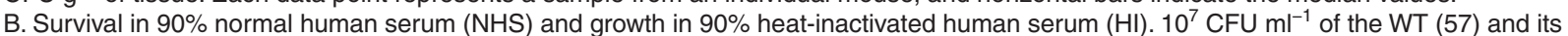
derivative strains were incubated in either $90 \%$ of NHS and $\mathrm{HI}$, and the number of CFUs was quantified by CFU enumeration on LB agar every hour. Since no CFUs were recovered from strains $\Delta a / e 2$ and $\Delta a / e 2 \mathrm{eV}$ incubated with $90 \%$ NHS, no statistical tests were performed. The dashed line corresponds to the limit of detection. Results are presented as the mean values and standard deviations of three independent experiments (B). Statistical significance was calculated by the Mann-Whitney test (A) $\left(^{* \star *}, P<0.0005\right.$; ${ }^{* * \star *}, P<0.0001$; NS, not significant) and by the Student's $t$-test (B, HI) (NS, not significant). Abbreviations: 57:WT; eV: empty vector (pABBR_Km); compl.: complemented (pABBR_Km-a/e2). [Colour figure can be viewed at wileyonlinelibrary.com] 
tested in our murine model of bacteremia. Compared to the WT strain, at $24 \mathrm{~h}$ post-inoculation, both mutants had significant fitness defects in the spleen, liver and kidneys, with a greater fitness defect attributed to gna (Fig. S1). Complementation of the gna mutation was achieved by cloning the gna gene, with its native promoter, into the complementing vector pABBR_Km (Crépin et al., 2018), creating plasmid pABBR_Km-gna and transforming into the gna mutant strain. Complementing the gne2 mutation was accomplished by fusing the coding sequence of gne2 with the promoter found upstream of $g n a$, and by cloning the fusion into the complementing plasmid pABBR_Km (Crépin et al., 2018), creating plasmid pABBR_Km-gne2 and transforming into the gne2 mutant strain. Although complementation of the single mutations did not fully restore the WT phenotype in vivo, the complemented strains colonized the spleen, liver and kidneys at a level that is significantly higher than their corresponding mutant (Fig. S1).

To determine whether the in vivo fitness defect of the three mutants was due to an overall growth defect, growth curve analyses were performed in Lysogeny Broth (LB) as well as in M9 minimal medium supplemented with $0.4 \%$ glucose and $0.2 \%$ casamino acids. By comparing the area under the curve (Todor et al., 2014) of the mutant strains with the WT in LB broth, a slight growth defect was observed in the $\Delta g n a$ and $\Delta a / e 2$ mutants ( -1.3 -fold), while no difference was observed for the $\Delta g n e 2$ mutant (Fig. S2A). A similar trend was observed in M9 minimal medium, with only a slight reduction in growth kinetics for all mutant strains ( $\Delta$ gna: -1.4 -fold; $\Delta$ gne2: -1 .3-fold; $\Delta a$ al e2:-1.5-fold) (Fig. S2B). Hence, we can conclude that the severe in vivo fitness defect of the mutant strains is not simply due to a reduced ability to proliferate.

As mutant strains are severely attenuated in our murine model of bloodstream infection, we sought to determine whether they were more susceptible to the bactericidal activity of serum. To test this hypothesis, $10^{7} \mathrm{CFU} \mathrm{ml}{ }^{-1}$ were incubated with $90 \%$ of active human serum and the number of surviving CFU was monitored for a period of $3 \mathrm{~h}$. As early as $1 \mathrm{~h}$ post-inoculation, no CFU were recovered from the $\Delta \mathrm{a} / \mathrm{e} 2$ mutant, while complementation of the mutation restored the WT level of serum resistance (Fig. 2B, NHS). To confirm that the increased susceptibility of $\Delta a / e 2$ to active human serum was mediated by the bactericidal activity of the complement, rather than an inability to grow in serum, the strains were incubated in $90 \%$ heat-inactivated serum. At $3 \mathrm{~h}$ post-inoculation, no difference in viability was observed between the strains (Fig. 2B, HI), confirming that the increased susceptibility of $\Delta \mathrm{a} / \mathrm{e} 2$ to human serum is indeed mediated by complement. The same phenotypes were observed in the single $\Delta$ gna and $\Delta$ gne2 mutants (Fig. S3). Together, these results demonstrate that gna and gne2 are required to resist the bactericidal activity of serum and may explain, at least in part, their severe fitness defect during bloodstream infection.

\section{Gna-Gne2 are required for capsule production, biofilm formation and motility}

Capsule production is directly associated with A. baumannii pathobiology, notably by promoting evasion of the host immune defenses, enhancing antimicrobial resistance and protecting cells in harsh environments (reviewed in (Singh et al., 2019)). Since previous studies have shown that UDP-GalNAcA is a component of the capsule in some strains of A. baumannii (Russo et al., 2013; Kenyon et al., 2016; Shashkov et al., 2017; 2018), we sought to determine if the increased susceptibility of the mutant strains to human serum was associated with a defect in capsule production. Capsule production by the mutant strains was assessed microscopically using Maneval's staining, and by extracting capsular polysaccharides. The smooth, uniform capsule produced by the WT is represented by the clear zone surrounding the cells, while no capsule was observed in the a/e2 mutant (Fig. 3A). Complementation of the a/e2 mutation restored capsule production to the WT level (Fig. 3A), confirming the role of gna-gne2 in capsule production. The same phenotypes were observed in the single gna and gne2 mutants, where no capsule was observed, and complementing the $\Delta g n a$ and $\Delta g n e 2$ strains with their corresponding gene in trans restored the WT phenotype (Fig. S4A).

To confirm the microscopy results, capsular polysaccharides were extracted, fractionated by electrophoresis, and visualized by Alcian blue staining (Geisinger and Isberg, 2015; Tipton and Rather, 2019). Extracted capsular polysaccharides from the WT strain appeared as a smear (Fig. 3B, lane 2). As expected, no capsular polysaccharides were extracted from the double a/e2 mutant (Fig. 3B, lane 3), while complementation of the a/e2 mutation restored capsular polysaccharide production to the WT level (Fig. 3B, lane 4). In addition to the loss of capsule production, the bacterial pellet of the $\Delta a / e 2$ strain was 'stickier' and more compact compared to the WT (Fig. S4C), which is in agreement with its loss of capsule production. Interestingly, inactivation of a/e2 seems to increase production of poly- $N$-acetylglucosamine (PNAG) (Fig. 3B, lane 3, upper band), a polysaccharide involved in biofilm formation (Choi et al., 2009). Not surprisingly, the same phenotypes were observed in the $\Delta g n a$ and $\Delta g n e 2$ mutants, where the absence of capsular polysaccharides resulted to a 'stickier' and more compact bacterial pellet; and seems to be associated with an increase production of PNAG (Fig. S4B). Accordingly, capsule and PNAG seem to be inversely regulated as a wZC mutant (capsule deficient) 
A

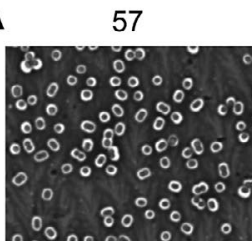

$\Delta a / e 2$

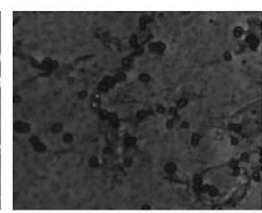

$\Delta \mathrm{a} / \mathrm{e} 2 \mathrm{compl}$

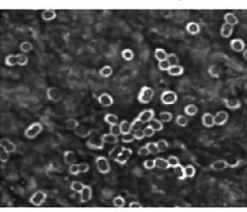

B

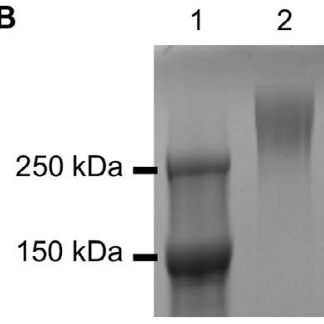

1. Ladder

2. 57
34

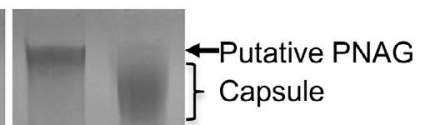

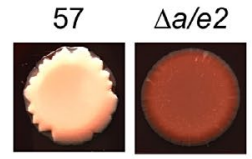

$\Delta a / e 2$ compl.
D

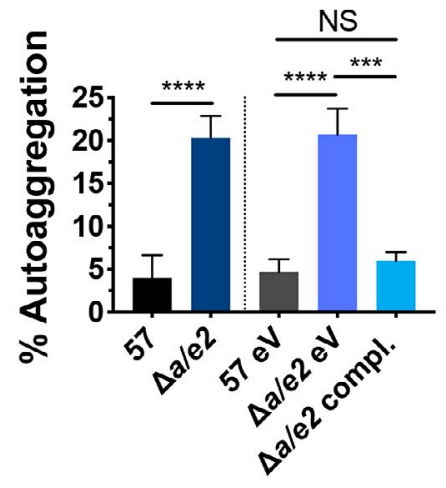

E
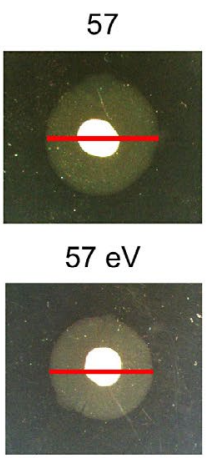

$\Delta a / e 2$

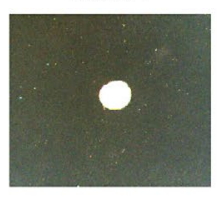

$\Delta a / e 2 \mathrm{eV}$

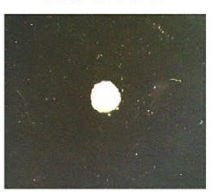

$\Delta a / e 2$ compl.

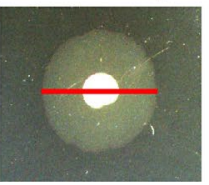

$\mathbf{F}$

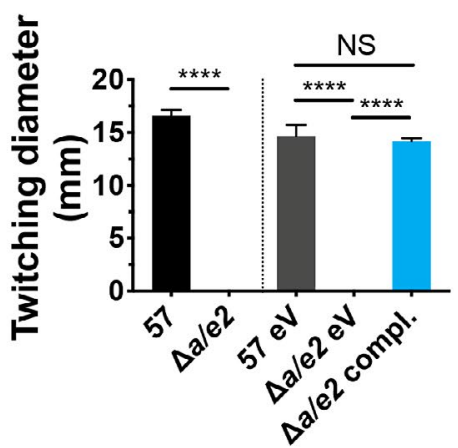

Fig. 3. Gna-Gne2 (A/E2) are required for capsule biosynthesis.

A. Microscopic analysis of capsule production by Maneval's staining of bacteria cultured for $24 \mathrm{~h}$ on LB agar plate. Images are representative of three independent experiments.

B. Analysis of the polysaccharides from lysates of bacteria cultured for $24 \mathrm{~h}$ on LB agar plate by SDS-PAGE and stained with $0.1 \%$ Alcian blue (section of the gel corresponding to the capsular polysaccharides). Images are representative of three independent experiments.

C. Colony morphology of the strains cultured on BHI CR plates for $48 \mathrm{~h}$ at $37^{\circ} \mathrm{C}$. Images are representative of three independent experiments.

D. Autoaggregation assay. Cultures of the WT (57) and its derivative strains were standardized in $10 \mathrm{ml}$ of LB to an $\mathrm{OD}_{600}$ of 2.0 in a culture tube and incubated statically at $37^{\circ} \mathrm{C}$ for $4 \mathrm{~h}$. A $200-\mu \mathrm{l}$ sample was taken about $0.5 \mathrm{~cm}$ below the surface at time 0 and $4 \mathrm{~h}$ p.i. for $\mathrm{OD}_{600}$ measurement. The percent autoaggregation was determined by dividing the $\mathrm{OD}_{600}$ value of the aggregated cells by the $\mathrm{OD}_{600}$ value of a control growth tube. E. Twitching motility. Strains were grown to an $\mathrm{OD}_{60}$ of 2.0 and an aliquot was stabbed to the bottom of a $1 \%$ EIKEN agar plate and incubated at $37^{\circ} \mathrm{C}$ for $18 \mathrm{~h}$. The red bars mark the twitching diameter. Images are representative of three independent experiments. Images are representative of three independent experiments.

F. Quantification of the twitching diameter (in $\mathrm{mm}$ ) from three independent experiments. Results are presented as the mean values and standard deviations of three independent experiments. Statistical significance was calculated by the one-way ANOVA with Tukey's multiple comparisons test $(\mathrm{D}$ and $\mathrm{F}){ }^{* * *}, P<0.005 ;{ }^{* * *}, P<0.001$; NS, Not significant). Abbreviations: 57 : WT; eV: empty vector (pABBR_Km); compl.: complemented (pABBR_Km-a/e2).

(Whitfield, 2006; Tipton et al., 2018; Singh et al., 2019)) of strain AB5075 also produced more PNAG than its corresponding WT strain (Fig. S4B).

Since the mutant strains appear to produce more PNAG than the WT strain, we then tested whether they were more prone to form a biofilm. Since $A$. baumannii is a strict aerobe, and autoaggregation was increased in our mutant strains (see paragraph below), an attempt to use the classical crystal violet binding assay to quantify biofilm formation failed. Instead, biofilm formation was indirectly evaluated using the Congo Red (CR) binding assay (Freeman et al., 1989). CR has been shown to bind to components of a biofilm, such as amyloid fibers, cellulose and PNAG, and can be used as a marker for biofilm formation. When cultured on a CR plate, a biofilm former will turn red and, occasionally, appears dry and wrinkly 
(referred to the red, dry and rough (rdar) morphotype). A non-biofilm former will not adopt that phenotype (Romling et al., 1998). Considering the potential increase in PNAG production by the a/e2 mutant, it was not surprising to observe that the $\Delta a / e 2$ mutant developed a rdar-like morphotype on $\mathrm{CR}$ plates under conditions typically unfavorable to biofilm formation (Fig. $3 \mathrm{C})\left(37^{\circ} \mathrm{C}\right.$, unpublished data and (De Silva et al., 2018)). Complementation of the $\Delta a / e 2$ mutant restored the WT phenotype, confirming the role of gna-gne2 in biofilm formation. Similarly, the single gna and gne2 mutants both adopted an rdar-like morphotype, where a stronger phenotype is observed in the $\Delta$ gna mutant (Fig. S4D). Complementation of both single mutations restored the WT phenotype. Similarly, a $\triangle$ wZC mutant of strain AB5075 adopted an rdar-like morphotype, suggesting that capsule production and biofilm formation are inversely regulated (Fig. S4D).

Autoaggregation is defined as the flocculation and settling of cells from a liquid static culture to the bottom of a culture tube (Diderichsen, 1980). Since autoaggregation and biofilm formation have been associated (Schembri et al., 2003; Charbonneau et al., 2006; Valle et al., 2008), we tested whether the mutant strains were more autoaggregative. Following static incubation at $37^{\circ} \mathrm{C}$ for $4 \mathrm{~h}$, we observed that $20.3 \%$ of the a/e2 mutant suspension autoaggregated, while only $4.0 \%$ of the WT strain did (5.1-fold increase in the mutant) (Fig. 3D). Complementing the a/ e2 mutation in trans restored the WT phenotype. Both gna and gne2 contributed to the autoaggregation phenotype, where gna had a greater contribution (20.7\% for $\Delta$ gna and $14.7 \%$ for $\Delta$ gne2) (Fig. S4E-F). Additionally, complementing both mutations in trans restored the WT phenotype.

In Escherichia coli, and other bacterial species, it has been demonstrated that adherence, autoaggregation and motility are reciprocally regulated (Ulett et al., 2006; Lane et al., 2007). Indeed, it would be counterproductive for a bacterium to adhere to a substrate and try to relocate at the same time. Thus, we can hypothesize that an adherent bacterium, such as the a/e2 mutant, should not be highly motile and vice versa. Twitching motility, a type of locomotion employed by $A$. baumannii, is defined as the migration of bacteria at the medium-plastic interface of a solid medium (agar), and is governed by the type IV pili (Mattick, 2002). Earlystationary phase cells $\left(\mathrm{OD}_{600}=2.0\right)$ were used to stab a twitching motility plate to the bottom of the petri dish. Following incubation at $37^{\circ} \mathrm{C}$ for $18 \mathrm{~h}$, the diameter of motile cells from the original stab was measured. As expected, inactivation of gna-gne2 completely suppressed twitching motility, while complementing the a/e2 mutation in trans restored the WT phenotype (Fig. 3E and F). Unlike the connection between PNAG production and biofilm formation, inhibition of twitching motility is not associated with capsule production, as no twitching defect had been observed in the AB5075 $\triangle W Z C$ mutant (Fig. S5A and D). Accordingly, preliminary RNAsequencing analysis revealed that expression of the type IV pili is decreased in the double a/e2 mutant (data not shown). Although complete suppression of twitching motility is observed in the single gna mutant, the $\Delta g n e 2$ strain showed an intermediate phenotype (twitching diameter of $10.7 \mathrm{~mm}$ versus $16.6 \mathrm{~mm}$ for the WT strain) (Fig. S5), which is consistent with the results from the $\mathrm{CR}$ binding and autoaggregation assays.

Altogether, these results clearly demonstrate the role of gna-gne2 in capsule production and their associated phenotypes such as resistance to the bactericidal activity of serum, biofilm formation, autoaggregation and motility. Also, these results show that although both gna and gne2 are influencing these phenotypes, gna seems to play a predominant role, especially for biofilm formation, autoaggregation and motility.

\section{Gna-Gne2 are important to maintain cell envelope homeostasis}

It has been proposed that glycosylation of the cell surface stabilizes the bacterial OM and, therefore, is important for cell envelope integrity (Iwashkiw et al., 2012). To test whether interruption of UDP-GalNAcA biosynthesis affects cell envelope integrity, assays such as resistance to vancomycin, bile salts, polymyxin $B$ and hydrolysis of 5-bromo-4-chloro-3'-indolyphosphate p-toluidine (XP) were performed. Since Gram-negative bacteria are intrinsically resistant to vancomycin, because it is too large to cross the $\mathrm{OM}$, we hypothesized that if gna-gne2 are important for cell envelope integrity (permeability), the $\Delta a / e 2$ mutant would be more susceptible to vancomycin than the WT strain. Using E-test strips (Biomerieux), we observed that the minimum inhibitory concentration (MIC) of the double a/e2 mutant was 133.8-fold lower than the WT strain (Fig. 4A). Gram-negative bacteria are also intrinsically resistant to detergent-like compounds such as bile salts, and mutants with an altered OM are more susceptible to these compounds (Hancock, 1984; Lamers et al., 2015). Accordingly, inactivation of gna-gne2 drastically compromises resistance to bile salts as the $\Delta a / e 2$ mutant was not able to growth on MacConkey agar, while the WT strain was unaffected (Fig. 4B). This growth inhibition is not associated with impaired capsule production since no difference in growth was observed between the AB5075 WT strain and its isogenic capsule $\triangle w Z c$ mutant (Fig. S6C). Therefore, we postulated that the severe susceptibility of the a/e2 mutant to bile salts is associated with an increase in membrane permeability. Hydrolysis of XP, a chromogenic substrate of the periplasmic alkaline phosphatase, is another approach to monitor alteration of the cell envelope integrity and OM permeability. 


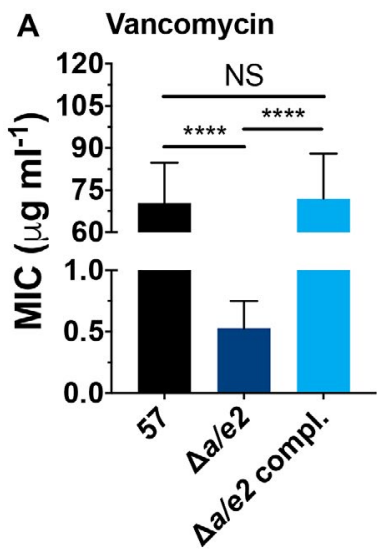

B
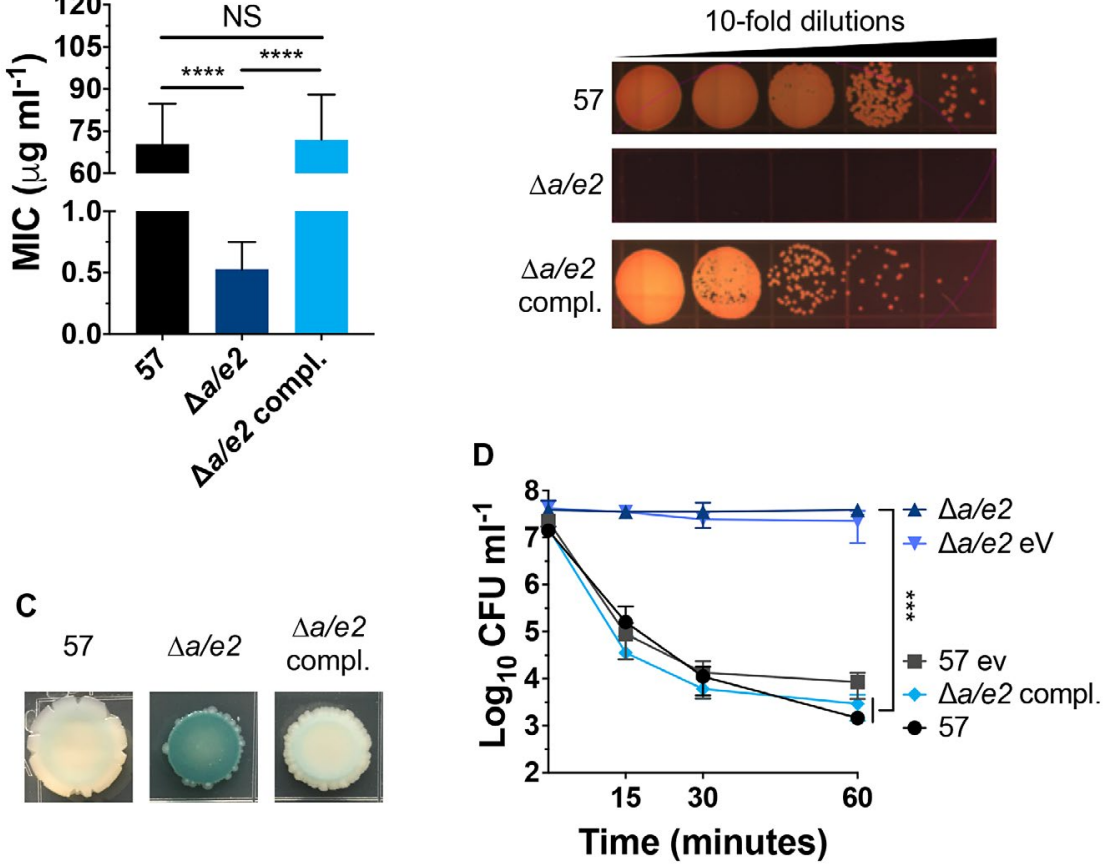

Fig. 4. The cell envelope integrity is altered in the double gna-gne2 (a/e2) mutant.

A. Minimal inhibitory concentration (MIC) of vancomycin on WT (57) and its derivative strains. MIC values were determined by E-test.

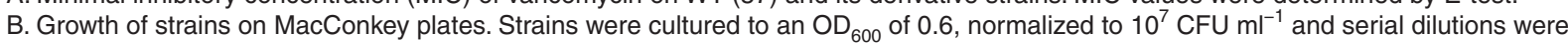
spotted on MacConkey plates. Images are representative of three independent experiments.

C. Colony morphology of strains grown on LB agar supplemented with BCIP-Toluidine (XP), a substrate of the periplasmic alkaline phosphatase PhoA. Images are representative of three independent experiments.

D. Survival in the presence of polymyxin B $\left(1 \mu \mathrm{g} \mathrm{ml}^{-1}\right)$. The number of surviving CFUs was quantified by CFU enumeration of LB agar at 15-, 30- and $60 \mathrm{~min}$ post-inoculation (p.i.). Results are presented as the mean values and standard deviations of three independent experiments. Statistical significance was calculated by the one-way ANOVA with Tukey's multiple comparisons test (A) $\left(^{\star \star \star}, P<0.005\right.$; NS, Not significant) and the two-way ANOVA with Tukey's multiple comparisons test (D) (*** $P<0.005)$. Abbreviations: 57 : WT; eV: empty vector (pABBR_Km); compl.: complemented (pABBR_Km-a/e2).

Karalewitz and Miller (2018) and Kamischke et al. (2019) have shown that when cultured on LB agar supplemented with XP, WT strains of $A$. baumannii remain white, while mutants with altered OM permeability hydrolyze the compound and present the blue phenotype. Accordingly, when grown on LB agar supplemented with XP, the WT strain remained white, while inactivation of gna-gne2 led to the blue phenotype (Fig. 4C).

Polymyxin B, a cationic polypeptide, binds to the negatively charged lipid $A$ and kills the bacterium by altering the OM permeability. Since we previously demonstrated that an increase in the cell envelope permeability increases susceptibility to polymyxin B (Crépin et al., 2018), we tested whether the a/e2 mutant exhibited enhanced susceptibility to $1 \mathrm{\mu g} \mathrm{ml}^{-1}$ polymyxin B. Surprisingly, the $\Delta \mathrm{a} /$ e2 mutant was highly resistant to concentrations of polymyxin B that were detrimental to WT (Fig. 4D), suggesting modification of LOS composition in the $\triangle \mathrm{a} / \mathrm{e} 2$ mutant (see next section). The a/e2 mutant appeared unaffected by exposure to $1 \mathrm{\mu g} \mathrm{ml}^{-1}$ polymyxin $\mathrm{B}$ as the number of CFU recovered at $60 \mathrm{~min}$ post-incubation was equivalent to those at time zero $\left(3.9 \times 10^{7}\right)$, while the WT strain was 4.0-logs more susceptible (Fig. 4D). Complementing the a/e2 mutation in trans restored the WT phenotype in all of these experiments (Fig. 4), confirming the role of gnagne2 in maintenance of the cell envelope homeostasis.

We then sought to determine the independent contribution of gna and gne2 to cell envelope integrity. We observed that gna was a major contributor, while gne2 played a lesser role to this phenotype. Indeed, the gna mutant resembled the double $\Delta a / e 2$ mutant in all of the phenotypes tested (Figs S6A,C,D,E and 4), while no differences were observed between the $\Delta g n e 2$ mutant and the WT strain when challenged to vancomycin and polymyxin B (Fig. S6B and F), and slight differences were observed in terms of growth on MacConkey agar (about 1-log growth defect) and hydrolysis of XP (Fig. $\mathrm{S} 6 \mathrm{C}$ and D). Complementation of the gna and gne2 mutation restored the WT phenotypes (Fig. S6). Together, these results present evidence that the UDP-GlaNAcA biosynthesis genes gna-gne2 are important to maintain cell envelope integrity and permeability. The results also 
demonstrated that cell envelope homeostasis is predominantly connected to expression of gna.

\section{Gna-Gne2 influences the composition of lipid A}

Although inactivation of gna-gne2 perturbs the cell envelope integrity, the $\Delta a / e 2$ and $\Delta$ gna mutants remain resistant to polymyxin B (Figs $4 \mathrm{D}$ and S6E). Since this bactericidal molecule destabilizes the $\mathrm{OM}$ by binding to lipid $\mathrm{A}$, we hypothesized that the gna-gne2 genes might play a role in the biosynthesis or modification of the lipid A. To test this hypothesis, cell-associated polysaccharides and LOS were extracted and fractionated by SDS-PAGE (Davis and Goldberg, 2012; Geisinger and Isberg, 2015; Tipton and Rather, 2019). In addition to extracting capsular polysaccharides, extraction of cell-associated polysaccharides also captures LOS (Iwashkiw et al., 2012; Geisinger and Isberg, 2015; Tipton and Rather, 2019). In the a/e2 mutant, the band corresponding to LOS was larger and more intense than the WT and the complemented strain, suggesting LOS modification(s) (Fig. 5A). These results were also confirmed by fractionating the LOS extracts by SDSPAGE electrophoresis (Fig. S7) (Davis and Goldberg, 2012). Compared to WT and the complemented strain, a faint upper band (lipid $A$ and core) and a bright lower band (lipid $A$ and truncated core) were observed in the a/e2 mutant (Fig. S7) (Geisinger and Isberg, 2015).

Next, using mass spectrometry, we determined the role of gna-gne2 in the biosynthesis and modification of lipid A. Lipid A extracts from the WT, $\Delta a / e 2$ and complemented strain were analyzed by a matrix-assisted laser desorption ionization-time of flight (MALDI-TOF) mass spectrophotometer in the negative ion mode (Pelletier et al., 2013). As expected, mass spectra of lipid A isolated from the $\Delta a / e 2$ mutant showed differences from that of the WT strain. Lipid A from the WT strain was predominantly decorated by the bis-phosphorylated, and hydroxylated, hexa- and hepta-acyl species, corresponding to $m / z 1,728$ and $m / z 1,910$, respectively (Fig. $5 B$; representative lipid A structures in Fig. $5 E$ and F). Conversely, the relative abundance in the mass spectra of lipid A from the a/e2 mutant showed reduced hydroxylated hexa-acyl species $(\mathrm{m} / z 1,728)$ and an increased in the hepta-acylated species $(m / z 1,894)$. This shift $(\Delta m / z 16)$ represents the loss of hydroxylation on both the hexa- and hepta-acylated species (shown as $-\mathrm{OH}$ in Fig. 5C and as a red arrow in Fig. 5E and F). We also observed a reduction in ion $\mathrm{m} / \mathrm{z} 1,882$, suggesting the replacement of a laurate $(\mathrm{C} 12)$ by a myristate (C14) on the hepta-acylated lipid A structure $(\mathrm{m} / \mathrm{z}$ $1,910)$. These species have been described elsewhere (Dixon and Darveau, 2005; Shaffer et al., 2007; Boll et al., 2015; Bartholomew et al., 2019; Leung et al., 2019). Complementation of the a/e2 mutation restored lipid $A$ biosynthesis and modifications to the WT level (Fig. 5D), indicating that in A. baumannii, gna-gne2 are involved, through an unknown mechanism, in the biosynthesis of lipid $A$.

The cell surface of $A$. baumannii is dominated by the hepta-acyl lipid A (Boll et al., 2015). It was postulated that the presence of the hepta-acyl lipid A fortifies the OM and confers resistance to cationic antimicrobial peptides and desiccation (Boll et al., 2015). In Enterobacteriaceae, it has also been observed that hepta-acylated lipid $A$ increases the cell envelope hydrophobicity (Bishop, 2005). Given that the $\Delta a / e 2$ mutant is more resistant to polymyxin B and produces more hepta-acylated lipid A, we hypothesized that the surface of the a/e2 mutant is more hydrophobic than that of the WT strain. To test this hypothesis, the bacterial adhesion to hydrocarbons (B.A.T.H) assay was performed (Rosenberg et al., 1980), where the WT, $\Delta a / e 2$ and complemented strains were suspended and incubated in $25 \%$ hexadecane for $15 \mathrm{~min}$ at room temperature (Goldberg et al., 1990). In this assay, higher hydrophobicity is indicated by a lower number of CFUs recovered from the aqueous phase. As hypothesized, the number of CFUs recovered in the aqueous phase from the a/e2 mutant was 2.0-logs less than the WT strain, suggesting that the cell surface of the a/e2 mutant is more hydrophobic. Similar results were also observed when the strains were incubated with $25 \%$ $n$-octane (Fig. S8A). In both conditions, complementing the a/e2 mutation restored the WT phenotypes (Figs 6A and $\mathrm{S} 8 \mathrm{~A}$ ), indicating that gna-gne2 modulate the cell envelope hydrophobicity.

Next, the independent contribution of gna and gne2 to the cell surface hydrophobicity were determined. In presence of hexadecane, the $\Delta g n a$ and $\Delta g n e 2$ mutants were, respectively 180.0 - and 8.9-times more hydrophobic than the WT strain (Fig. S8B-C). Complementing both gna and gne2 mutations in trans restored the WT phenotype in both conditions, confirming their role in influencing the cell surface hydrophobicity.

As inactivation of gna-gne2 altered the cell envelope integrity and lipid $A$ biosynthesis, we tested whether the envelope stress response (ESR) was induced in the double a/e2 mutant. The ESR senses environmental changes and stresses, and stimulates the cell's adaption to restore the cell envelope homeostasis (reviewed in (Mitchell and Silhavy, 2019)). Expression of the $A$. baumannii ESR genes ( $d s b A$, degP, baeR, $r s t A$ ) (Crépin et al., 2018) was quantified by qRT-PCR. Compared to the WT strain, expression of all four genes was induced (dsbA: 9.1-; degP: 23.3-; baeR: 13.0-; and rstA: 14.7fold) in the a/e2 mutant (Fig. 6B). Complementation of the a/e2 mutation restored expression of ESR genes to the WT level, confirming that inactivation of gna-gne2 induced the ESR. 

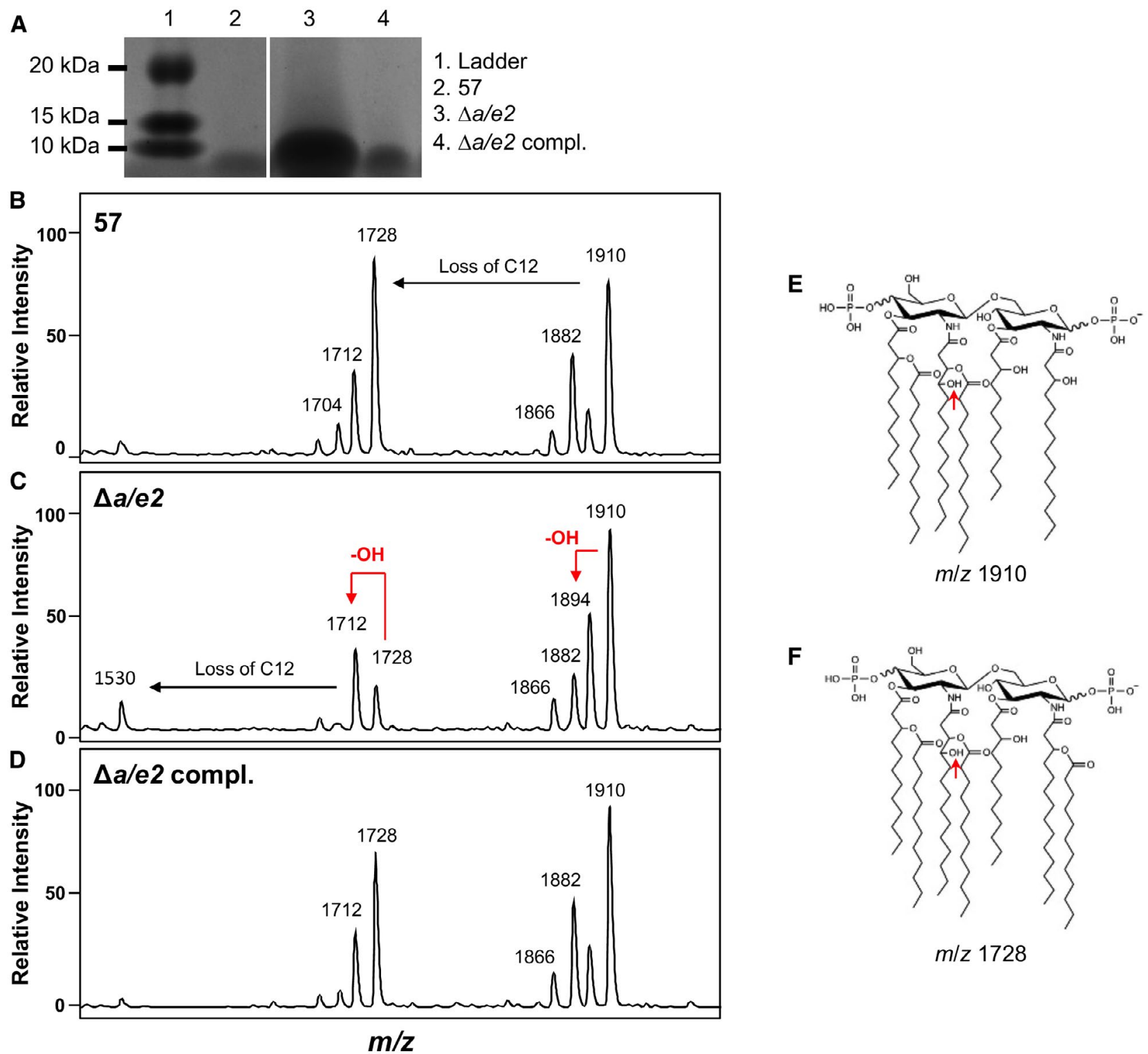

$m / z 1728$

Fig. 5. Inactivation of gna-gne2 (a/e2) affects the composition of lipid A.

A. Analysis of polysaccharides from lysates of bacteria cultured for $24 \mathrm{~h}$ on LB agar plate by SDS-PAGE and stained with $0.1 \%$ Alcian blue (section of the gel corresponding to the LOS band).

B-D. MS analysis of the lipid A from the WT (B), $\Delta a / e 2$ (C) and complemented strain (D). Mutant strain lipid A (C) loses hydroxylation (OH, observed as $\Delta m / z 16$, red arrows; mediated by the enzyme LpxO), which is restored in complemented strain (D). Additional loss of C12 to generate penta-acylated lipid A ( $\mathrm{m} / \mathrm{z}$ 1530) is likely a result of the harsh lipid A extraction conditions. Mass differences of $\Delta \mathrm{m} / \mathrm{z} 28$ (from $\mathrm{m} / \mathrm{z}$ 1910 to $\mathrm{m} / \mathrm{z} 1882$ ) and $\Delta \mathrm{m} / \mathrm{z} 16$ (from $\mathrm{m} / \mathrm{z} 1910$ to $\mathrm{m} / \mathrm{z} 1894$; and from $\mathrm{m} / \mathrm{z} 1728$ to $\mathrm{m} / \mathrm{z} 1712$ ) are attributed to acyl chain length heterogeneity and hydroxylation status, respectively.

E-F. Predicted structure of $m / z 1910(E)$ and $m / z 1728(F)$, with the site of hydroxylation indicated in red. Images and $m / z$ spectra are representative of three independent experiments. Abbreviations: 57: WT; compl.: complemented (pABBR_Km-a/e2).

Although independent inactivation of gna and gne2 induced the ESR (Fig. S8D-E), we observed that its induction is primarily associated with gna. Indeed, the ESR expression pattern in the single gna mutant was similar to the double a/e2 mutant (dsbA: 3.6-; degP: 16.0-; baeR: 8.3-; and rstA: 7.6-fold), while inactivation of gne2 slightly stimulated the ESR (dsbA: 2.3-; degP: 2.7-; baeR: not differentially expressed; and rstA: 2.1-fold) (Figs S8D-E and 6B). Complementing the mutations in trans restored ESR gene expression to the WT level, demonstrating the role of gna and, to a lesser extent gne2, in modulating the ESR.

Together, these results demonstrated that the UDPGalNAcA biosynthesis genes gna-gne2 are modulating 

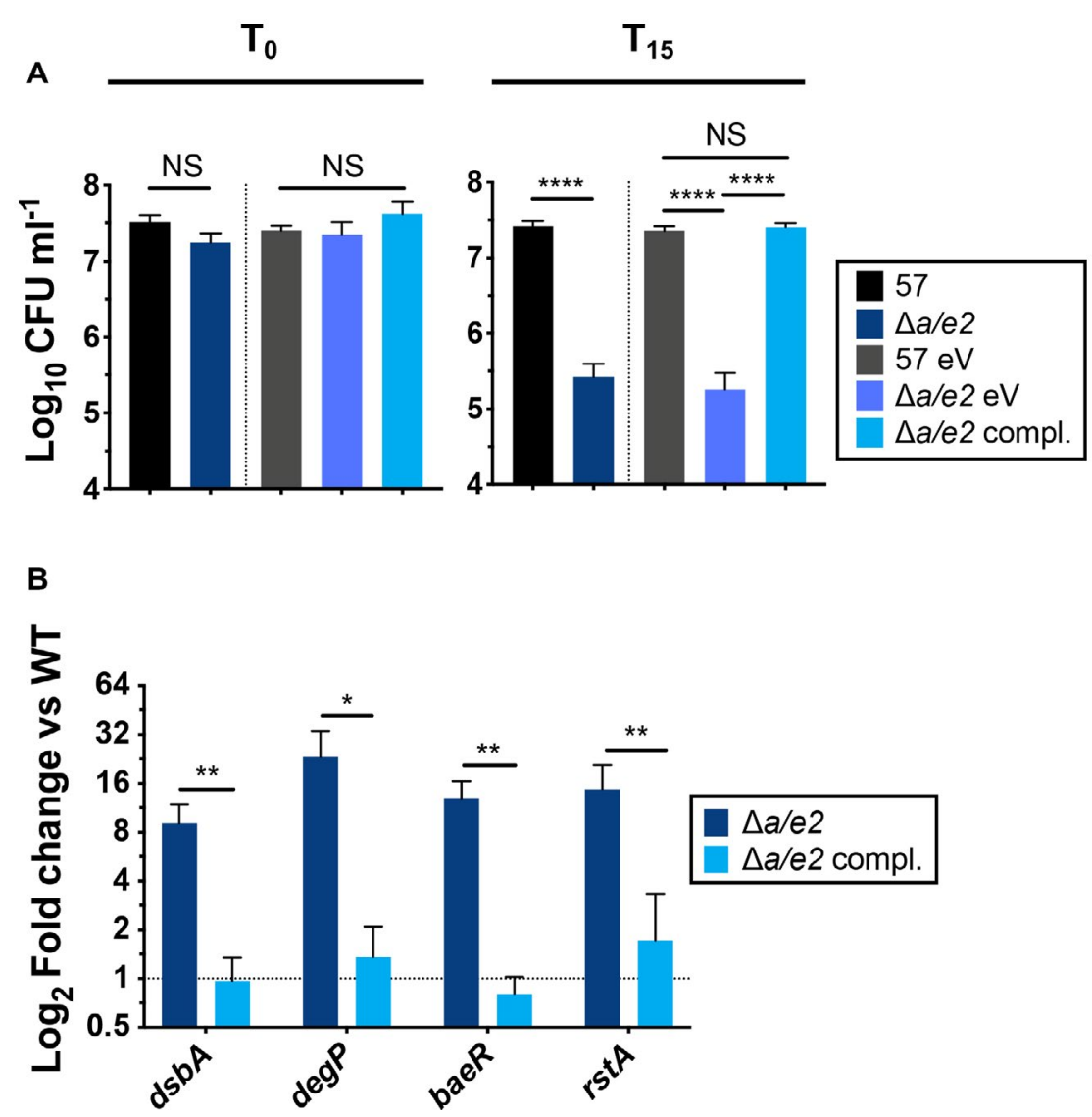

Fig. 6. Inactivation of gna-gne2 (a/e2) increases the cell envelope hydrophobicity and induces the envelope stress response. A. Bacterial adherence to hydrocarbons. $10^{7} \mathrm{CFU} \mathrm{ml}{ }^{-1}$ were incubated in the presence of $25 \%$ hexadecane, and the number of CFUs from the aqueous phase were recovered at $15 \mathrm{~min}$ post-inoculation. An increase in cell envelope hydrophobicity is represented by a lower number of CFUs recovered from the aqueous phase.

B. Expression of genes involved in the envelope stress response (ESR, dsbA, degP, baeR and $r s t A$ ) between the WT, $\triangle a / e 2$ and the complemented strain. Gene expression was evaluated by qRT-PCR and compared between the WT, $\triangle a / e 2$ and the complemented strain. The dashed line corresponds to the cutoff for a significant difference in expression. All results are the mean values and standard deviations of three independent experiments. Statistical significance was calculated by the one-way ANOVA with Tukey's multiple comparisons test (A) and by the Student's $t$-test (B) $\left({ }^{*}, P<0.05 ;{ }^{* *}, P<0.01 ;{ }^{* \star *}, P<0.0001\right.$; NS, not significant). Abbreviations: 57 : WT; eV: empty vector (pABBR_Km); compl.: complemented (pABBR_Km-a/e2).

the cell envelope homeostasis by affecting the composition of the lipid A, which influences cell surface hydrophobicity and the ESR. We also observed that these phenotypes were mostly modulated by gna.

\section{Gna-Gne2 are involved in antibiotic resistance}

In addition to providing structural integrity to the cell, the cell envelope also protects the bacterium from environmental stresses and antibiotics. Since we demonstrate that inactivation of gna-gne2 alters cell envelope homeostasis and increases cell surface permeability and hydrophobicity, we sought to determine whether gna-gne2 are important for antibiotic resistance. First, using E-test strips, we determined the MIC of the $\Delta a$ / e2 mutant to amoxicillin (penicillin, hydrophilic), imipenem (carbapenem, hydrophilic) and gentamicin (aminoglycoside, hydrophobic). As shown in Fig. 7A, the a/e2 mutant was more susceptible to all three antibiotics, where the MICs for amoxicillin, gentamicin and imipenem were, respectively, 4.8-, 8.4- and 10.9-fold lower than the WT strain. Second, we determined the percent survival of the WT, a/e2 mutant and complemented strain to a specific concentration of cefotaxime (50 $\mathrm{g} \mathrm{m} \mathrm{m}^{-1}$; cephalosporin, hydrophilic), carbenicillin (100 $\mu \mathrm{g} \mathrm{ml}^{-1}$; carboxypenicillin, hydrophilic) and tetracycline $\left(20 \mathrm{\mu g} \mathrm{ml}^{-1}\right.$; tetracycline, both hydrophobic and hydrophilic). Consistent with the MIC data, the a/e2 mutant was 12.1-, 6.9- and 3.2-times more susceptible to cefotaxime, carbenicillin and tetracycline, respectively (Fig. 7B). In trans complementation of the a/e2 mutation restored resistance to these antibiotics to WT levels (Fig. 7), confirming the role of gna-gne2 in modulating antibiotic resistance. 


\section{A Amoxicillin}

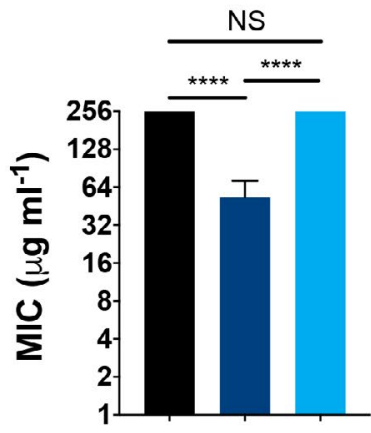

Gentamicin

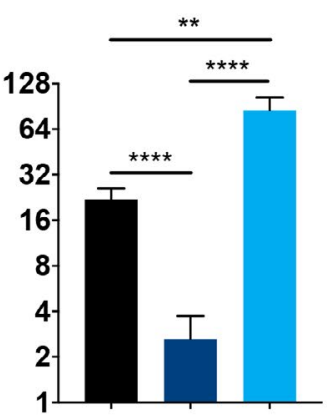

Imipenem

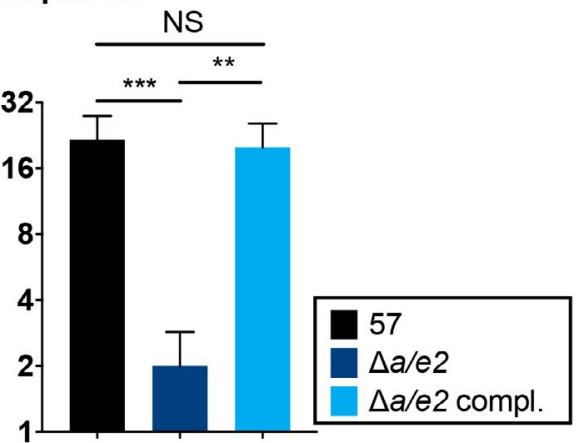

B Cefotaxime $(50 \mu \mathrm{g} / \mathrm{ml})$

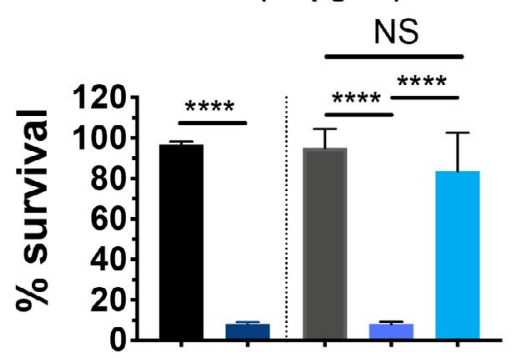

Carbenicillin $(100 \mu \mathrm{g} / \mathrm{ml})$

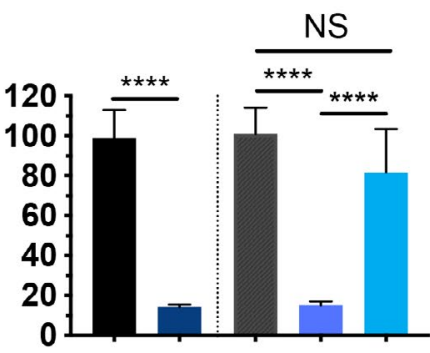

Tetracycline $(20 \mu \mathrm{g} / \mathrm{ml})$

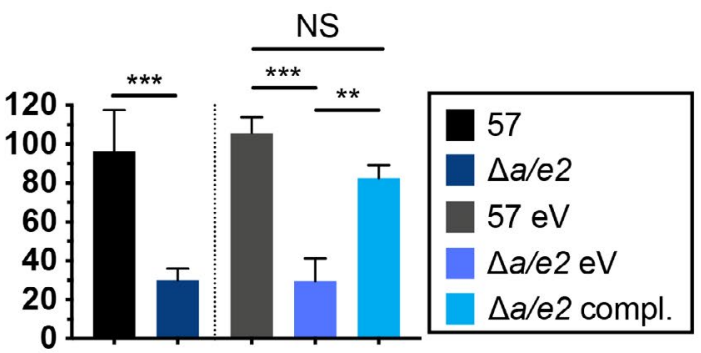

Fig. 7. Gna-Gne2 (A/E2) influence resistance to antibiotics.

A. Minimal inhibitory concentration (MIC) of the WT (57) and its derivative strains to different antibiotics. MIC values were determined by the E-test method.

B. Percent survival in the presence of a defined concentration of antibiotic. $10^{7} \mathrm{CFU} \mathrm{m}^{-1}$ were exposed to different antibiotics and the percent survival was determined by dividing the number of CFUs recovered from the antibiotic challenge by the number of CFUs recovered from the corresponding untreated sample. All results are the mean values and standard deviations of three independent experiments. Statistical significance was calculated by the one-way ANOVA with Tukey's multiple comparisons test $\left({ }^{* *}, P<0.01\right.$; ${ }^{* * *}, P<0.005$; ${ }^{* * *}, P<0.0001$; NS, not significant). Abbreviations: 57: WT; eV: empty vector (pABBR_Km); compl.: complemented (pABBR_Km-a/e2).

Similar to previous experiments, antibiotic resistance was primarily associated with gna (Fig. S9-10). Although the effect is less severe, inactivation of gna recapitulated the antibiotic resistance profile of the double a/e2 mutant (Figs S9A,B and 7), while inactivation of gne2 only slightly increased susceptibility to the antibiotics tested (Fig S10 and 7). Complementation of both gna and gne2 mutations restored the WT phenotype, indicating their influence on antibiotic resistance in $A$. baumannii. These results demonstrate the importance of the GalNAcA biosynthesis genes gna-gne2 in resistance to antibiotics in multidrug resistant $A$. baumannii.

Altogether, the results presented in this study demonstrated the crucial role of Gna-Gne2 in the pathobiology of A. baumannii, affecting capsule production, LOS biosynthesis and modification, envelope homeostasis, antibiotic resistance and in vivo fitness.

\section{Discussion}

With its high prevalence of infections, high mortality rate and increasing resistance to antibiotics, the WHO identifies $A$. baumannii as a top priority pathogen for which new antimicrobials are urgently needed (Lawe-Davies and Bennett, 2017; Willyard, 2017). Since the pathobiology of $A$. baumannii is poorly defined, the development of new preventive and therapeutic agents is considerably limited. Accordingly, the identification and characterization of $A$. baumannii genes that are required during infection will allow us to design strategies to prevent and treat infections caused by this notorious pathogen.

We and others have previously identified the full set of genes required during infection (Wang et al., 2014; Gebhardt et al., 2015; Subashchandrabose et al., 2016; Murray et al., 2017; Crépin et al., 2018). Among the genes identified, mutation of the UDP-GalNAcA biosynthesis genes gna-gne2 (also referred as $t v i B C$ ) resulted in a severe fitness defect during bloodstream infection (-199.4-fold for gna and -445.7-fold for gne2) (Crépin et al., 2018). In the current study, we defined the contribution of these two genes (Fig. 1A) to the pathobiology of $A$. baumannii. Indeed, we demonstrated that gna-gne2 are essential during bloodstream infection (Figs. 2 and $\mathrm{S} 1,3$ ) and for capsule and LOS synthesis (Figs. 2 and 5; and Figs. S4 and S7). These genes also modulate cell 
envelope homeostasis (Figs. 3-6 and S4-8) and resistance to antibiotics (Figs. 7 and S9-10).

An in silico analysis revealed that Gna-Gne2 of $A$. baumannii are highly homologous to Gna-Gne2 of $P$. aeruginosa and $S$. Typhi (Table S1), enzymes known to synthesize UDP-GalNAcA (Zhang et al., 2006). Furthermore, based on genetic analysis and published capsule structures of different strains of $A$. baumannii, presence of UDP-GalNAcA in the capsule composition is associated with gna-gne2 (Russo et al., 2013; Kenyon et al., 2016; Shashkov et al., 2017; 2018). However, in $A$. baumannii, no genetic data had previously confirmed this association. Herein, we demonstrated that inactivation of gna-gne2 not only reduced the amount of capsule present at the cell surface, but abolished its synthesis (Figs. 3 and S4). Absence of capsule in the gna-gne2 mutant is also connected to its extreme susceptibility to the bactericidal activity of serum and may explain, at least in part, the severe colonization defect of the bloodstream by the a/e2 mutant (Figs. 2, 3 and S1).

Interestingly, in the a/e2 mutant, abolition of capsule synthesis appears to be associated with production of PNAG (Figs. 3B and S4B), which in turn favors biofilm formation (Figs. 3C and S4D). Furthermore, inactivation of gna-gne2, that is, suppression of UDP-GalNAcA biosynthesis, promotes bacterial autoaggregation (Figs 3D and S4E,F), which is associated with biofilm formation (Schembri et al., 2003; Charbonneau et al., 2006; Valle et al., 2008). It is possible that the absence of capsule unmasks adhesins at the cell surface which promote adhesion to both abiotic and biotic surfaces, as well as to other cells. In E. coli and other species, it was observed that the capsule could shield the function of the adhesin Ag43 and the autotransporter AIDA-1 (Schembri et al., 2004). Similarly, autoaggregation was enhanced in a capsule mutant of $A$. baumannii strain ATCC17978 (Lees-Miller et al., 2013). Based on these observations, we were interested to visualize, by transmission electron microscopy, whether the a/e2 mutant was presenting more adhesins at its cell surface than the WT strain. Unfortunately, negative staining using phosphotungstic acid failed to properly stain the bacteria and, even less, its surface appendages. The faint staining of the a/e2 mutant could be explained by the reduced charge and the increased hydrophobicity of its cell surface (Figs. 6A and S8A-C).

Capsule biosynthesis and protein glycosylation are connected as they are synthesized by an en bloc mechanism (Whitfield and Paiment, 2003; Lees-Miller et al., 2013). In A. baumannii, a bifurcated pathway has been proposed, where the common pathway includes synthesis of the nucleotide-activated monosaccharides followed by their transfer, by the action of multiple glycosyltransferases (such as ItrA/PgIC), onto an undecaprenolphosphate (Und-P) lipid carrier at the cytoplasmic face of the inner membrane. The complex (Und-P-linked repeat units) is then flipped to the periplasmic space by the oligosaccharide-unit translocase Wzx (Whitfield, 2006; Lees-Miller et al., 2013; Geisinger et al., 2019). Once in the periplasmic space, the two pathways diverge, where the capsular polysaccharides are polymerized and exported to the cell surface by the Wzy and Wzabc machinery (Whitfield, 2006), while the glycans used in O-glycosylation are transferred to proteins on the cell surface by the phosphoglycosyltransferase PgIC (Lees-Miller et al., 2013). This has been shown in A. baumannii strain ATCC17978 by Lees-Miller et al. (Lees-Miller et al., 2013), where the same glycan can be used for both capsule synthesis and protein glycosylation.

Our genetic approach identified gna-gne2 and consequently, UDP-GalNAcA, as being required to maintain the cell envelope integrity. Assays such as resistance to vancomycin, bile salts and polymyxin $B$, as well as hydrolysis of $\mathrm{XP}$, have been previously used to evaluate the integrity of the cell envelope (Lamers et al., 2015; Crépin et al., 2018; Karalewitz and Miller, 2018). The increased susceptibility to vancomycin and bile salts, hydrolysis of XP, and resistance to polymyxin $\mathrm{B}$ observed in the a/e2 mutant (Figs 4 and S6) strongly support this hypothesis. Since it has been proposed that glycosylation of cell surface proteins stabilizes the $\mathrm{OM}$ and regulates the integrity of the cell envelope, we speculate that in addition to being involved in capsule synthesis, UDP-GaINAcA might also be used as a substrate for glycosylating the cell surface proteins. These glycosylated proteins could provide protection from the host immune system, in a fashion similar to that proposed by Iwashkiw et al. (2012). It would be interesting to determine whether UDP-GalNAcA is indeed a glycan used in $O$-glycosylation in A. baumannii AB0057 and strains encoding gna-gne2. If so, the identification of such structures, as well as their contribution to the pathobiology of $A$. baumannii could be characterized.

Fractionation of extracted LOS, coupled with the characterization of lipid A by mass spectrometry (Figs 5 and S7), confirmed that gna-gne2 are indeed critical for modulating cell envelope homeostasis. The profile of lipid A from the a/e2 mutant differed from the WT strain and revealed a shift toward un-hydroxylated lipid $A$ as well as a shift in the relative proportion of hexa- versus hepta-acylated lipid $A$ specie. The increase in the relative abundance of the heptaacyl lipid $A$ in the OM of the a/e2 mutant is consistent with its resistance to polymyxin $B$ and its increased cell surface hydrophobicity. However, a quantitative analysis such as gas chromatography of the lipid $A$ is needed to confirm these observations.

It has recently been shown that hydroxylation of lipid $A$ is important for resistance to cationic antimicrobial peptides, survival within the blood, reducing the inflammatory response, and finally, in vivo fitness (Bartholomew et al., 
2019). In addition, Boll et al. (2015) have shown that heptaacylated lipid A stimulates the TLR-4 response. Based on these observations, we hypothesize that the a/e2 mutant (reduction of the hydroxy hexa-acyl and increase of the hepta-acyl species) triggers the immune response and could explain its severe in vivo fitness defect.

Lipopolysaccharide/lipooligosaccharide biosynthesis and protein glycosylation are connected by a similar en bloc synthesis pathway that was described between capsule and $O$-glycosylation. Indeed, for $P$. aeruginosa, it was shown that the same glycan can be used for both $\mathrm{O}$-antigen synthesis and protein glycosylation (Castric et al., 2001). In Campylobacter jejuni, UDP-GalNAc is a component of LOS, the capsule, and is involved in protein glycosylation. In $P$. aeruginosa strain $\mathrm{O6}$, it has been shown that the UDP-GalNACA is a component of the O-antigen (Knirel, 1990; Belanger et al., 1999; Lam et al., 2011). In A. baumannii, it has been reported that this sugar is involved in the biosynthesis of LOS in strains $\mathrm{O} 5$ and 24 (Haseley and Wilkinson, 1996; Vinogradov et al., 2003). Based on the modified fractionation of the LOS in the a/e2 mutant, where a shift in its molecular weight is observed (Figs. 5 and S7), we hypothesize that UDP-GalNAcA is a component of LOS in strain AB0057. It is possible that interrupting synthesis of UDP-GalNACA induces a compensatory mechanism by which other UDP-linked glycans are produced and incorporated into the LOS instead of UDP-GalNAcA. With the recent development of top down tandem mass spectrometric analysis of intact LOS (Oyler et al., 2018; Klein et al., 2019), it would be interesting to compare the LOS composition between the a/e2 mutant and the WT strain. Similarly, it is possible that the mutant strain is encountering an accumulation of UDP-linked glycans at its inner membrane cytoplasmic face, which could eventually affect the integrity of the cell envelope. This hypothesis could be verified by performing a metabolomic analysis between the WT and the a/e2 mutant.

The ESR has evolved to sense environmental insults (e.g immune system, temperature, $\mathrm{pH}$ and antibiotics) and monitor the defects or the damage to the cell envelope in order to restore its homeostasis (Mitchell and Silhavy, 2019). Accordingly, we were not surprised to observe that the ESR was induced in the a/e2 mutant and showed that Gna-Gne2 are key players in the maintenance of the cell envelope homeostasis. However, the mechanisms connecting gna-gne2 and the ESR are yet to be determined.

Finally, $A$. baumannii is categorized as a multi-, extremely- or pan-drug resistant, which makes it extremely difficult to treat. As a result, in some cases, no antimicrobial treatment options are available (Dijkshoorn et al., 2007). By understanding the pathobiology of $A$. baumannii, we will be able to identify novel targets for the development of new antimicrobials. Herein, we determined that gna-gne2 are required to resist antibiotics. The $\Delta a /$ e2 mutant is more susceptible to amoxicillin, gentamicin, imipenem, cefotaxime, carbenicillin and tetracycline (Figs 7 and S9-10). These results are in line with the phenotypes observed in the a/e2 mutant (inhibition of capsule synthesis and cell envelope integrity alteration) and open new avenues in the development of new therapeutics and strategies to treat the infections caused by $A$. baumannii. Indeed, by inhibiting the UDP-GalNAcA biosynthesis pathway, A baumannii not only becomes less pathogenic, but is also more susceptible to known antibiotics.

During the revision of this article, a study analyzing the role of gnaA (homolog of the gna described herein) in the physiology of the $A$. baumannii MDR strain MDR-ZJ06 was reported (Xu et al., 2019). Despite major differences in their K locus (AB0057 vs MDR-ZJ06), where wzX, qnr and a homolog of gna are the only genes in common from the variable region (Kenyon and Hall, 2013), the findings described by Xu et al. (2019) are in agreement with the results described in this report, reinforcing the idea that gna, and its homologs, play a pivotal role in the pathobiology of $A$. baumannii that goes beyond capsule production.

In summary, in this study we have characterized the role of gna-gne2 in the pathobiology of $A$. baumannii. We demonstrated that gna-gne2 are part of a complex network connecting capsule and LOS synthesis, cell envelope homeostasis, in vivo fitness and antibiotic resistance. In addition, the phenotypes observed in the a/e2 mutant are mostly attributed to gna, a highly conserved UDP- $N$ acetylglucosamine C- 6 dehydrogenase among $A$. baumannii strains.

\section{Experimental procedures}

\section{Ethics statement}

All procedures involving the use of mice were approved by the University Committee on Use and Care of Animals (UCUCA) of the University of Michigan Medical School (protocol \# PRO00007111), in accordance with the Institutional Animal Care and Use Committee (IACUC), the Office of Laboratory Animal Welfare (OLAW) and the United States Department of Agriculture (USDA). The protocol was accredited by the Association for Assessment and Accreditation of Laboratory Animal Care, International (AAALAC, Intl.) following the Guide for the care and use of laboratory animals of the National Research Council of the National Academies ( $8^{\text {th }}$ edition). Neutrophils were depleted by intraperitoneal injection of $500 \mu \mathrm{g}$ of rat anti-mouse monoclonal antibody (MAb) RB6-8C5 (RB6) (BioXCell) 24 hours prior infection (Conlan and North, 1994; van Faassen et al., 2007; Crépin et al., 2018). Mice were euthanized by inhalant anesthetic overdose followed by vital organ removal.

\section{Bacterial strains, plasmids and growth media}

Strains and plasmids used in this study are listed in Table S3. Herein, the WT strain is a $\mathrm{Km}$-sensitive derivative of the 
AB0057 strain (Crépin et al., 2018). Bacteria were cultured in LB at $37^{\circ} \mathrm{C}$. Bacteria were also cultured in M9 minimal medium supplemented with $0.4 \%$ glucose and $0.2 \%$ casamino acids. Antibiotics and reagents were added as required at the following concentrations: kanamycin, $50 \mathrm{\mu g} \mathrm{ml}^{-1}$; ampicillin, $100 \mathrm{\mu g} \mathrm{ml}^{-1}$; amikacin, $10 \mathrm{\mu g} \mathrm{ml}^{-1}$; sucrose, $10 \% \mathrm{w} \mathrm{v}^{-1}$; and $\mathrm{XP}, 100 \mathrm{\mu g} \mathrm{ml}^{-1}$.

\section{Construction of non-polar mutants and complemented strain}

Primers used in this study are listed in Table S4. Generation of in-frame markerless mutants was achieved by allelic exchange as described by Crépin et al. (2018). Briefly, the mutant alleles were constructed using the Gibson assembly (Gibson et al., 2009), where the $5^{\prime}$ end of the gene of interest (goi) to be deleted possessed at least $1 \mathrm{~Kb}$ including the initiation codon, while the $3^{\prime}$ region consisted of at least $1 \mathrm{~Kb}$ including the last 7 codons. The $5^{\prime}$ and $3^{\prime}$ regions were cloned into pCVD442_MCS_Km (Crépin et al., 2018), which results in the in-frame deletion of the internal region of the goi. The construct was transformed into the donor strain S17-1, and was transferred to the $\mathrm{AB} 0057^{\mathrm{Km}}$ (WT) strain by conjugation. Transconjugants were selected on LB agar containing kanamycin. Individual colonies were cultured $2 \mathrm{~h}$ in LB broth, diluted and spread on LB agar plates containing $10 \%$ (wt $\mathrm{vol}^{-1}$ ) sucrose to select the second recombination event. Sucrose-resistant and kanamycin-sensitive isolates were screened by PCR to confirm deletion of the goi.

Complementation of the gna-gne2 deletion was achieved by cloning the gna-gne2 region, including $~ 300$ nucleotides upstream of gna, in the pABBR_Km plasmid.Complementation of the gna deletion was accomplished by cloning, by Gibson assembly (Gibson et al., 2009), the gna gene, including 300 nucleotides upstream of it, in pABBR_Km. Complementation of the gne2 deletion was achieved as described for gna, with the exception that since gne 2 is in operon with gna, the promoter region upstream of gna was fused to the coding region of gne2 and cloned into pABBR_Km.

\section{Growth curves}

The growth of WT and its derivative strains was monitored in LB or M9 minimal medium supplemented with $0.4 \%$ glucose and $0.2 \%$ casamino. Strains were cultured overnight in LB, washed twice in PBS and the $\mathrm{OD}_{600}$ was adjusted to 0.01 in medium. Growth was measured by $\mathrm{OD}_{600}$ determination every 30 min with a BioScreen $\mathrm{C}$ Analyzer at $37^{\circ} \mathrm{C}$ with continuous shaking. The quantification of growth, or growth potential, and comparison between strains was performed by calculating the area under the curve of each growth curve (Tonner et al., 2015).

\section{Murine model of bacteremia}

All infections performed in this study were mono-infection. Infections were performed as described previously (Smith et al., 2010; Crépin et al., 2018), in which female CBA/J mice aged from 6- to 8-weeks-old were inoculated via tail vein injection with $10^{7} \mathrm{CFU}$. At 24 hours post-infection (hpi), mice were euthanized, and the spleen, liver and kidneys were aseptically removed, homogenized, diluted and plated on LB agar plates to determine the colonization level in these organs.

\section{Resistance to normal human serum}

Growth of $A$. baumannii in human serum was performed as previously described (Crépin et al., 2018). Briefly, bacteria were cultured overnight in LB broth at $37^{\circ} \mathrm{C}$. Bacterial cultures were resuspended 1:100 in fresh medium and grown to mid-log $\left(O D_{600}\right.$ of 0.6). Bacteria were washed with PBS and $10^{7} \mathrm{CFU} \mathrm{ml}{ }^{-1}$ were incubated either with $90 \%$ normal human serum or $90 \%$ heat-inactivated serum (Innovative Research). Suspensions were incubated at $37^{\circ} \mathrm{C}$ and viable cell counts were determined at $0,1,2$ and $3 \mathrm{~h}$ post-incubation on LB agar plates.

\section{Microscopic analysis of the capsule by Maneval's staining}

Capsule production was microscopically visualized using Maneval's staining method (Maneval, 1941). Briefly, the bacterial strains were grown $24 \mathrm{~h}$ on LB agar at $37^{\circ} \mathrm{C}$. One colony was mixed with a drop of $1 \% \mathrm{CR}$ and air-dried. The dried smear was then counterstained with Maneval's stain (0.05 $\% \mathrm{w} \mathrm{v}^{-1}$ acid fuchsin, $2.8 \% \mathrm{w} \mathrm{v}^{-1}$ ferric chloride, $3.2 \% \mathrm{v} \mathrm{v}^{-1}$ phenol and $4.68 \% \vee^{-1}$ glacial acetic acid). Excess stain was washed from the slide with a stream of water and dried with bibulous paper. Images were captured with a Zeiss Axioplan 2 epifluorescence microscope equipped with a 100x PlanNeofluor objective with a numerical aperture of 1.3 .

\section{Polysaccharide extraction, electrophoresis and visualization}

Surface polysaccharides were extracted as described by Tipton and Rather (2019). Samples were separated on SurePAGE, Bis-Tris, $4 \%-12 \%$ gel (GenScript) and stained for $60 \mathrm{~min}$ with $0.1 \%\left(w v^{-1}\right)$ Alcian blue. Gels were imaged with ChemiDoc ${ }^{\mathrm{TM}}$ Touch Imaging system (Bio-Rad). The top section of the gel corresponds to the capsular polysaccharides while the bottom section of the gel corresponds to the LOS (Geisinger and Isberg, 2015; Tipton and Rather, 2019).

\section{Congo red binding assay}

Bacteria were cultured overnight in LB broth at $37^{\circ} \mathrm{C}$. The cultures were then adjusted to an $\mathrm{OD}_{600}$ of 1.0 in brain heart infusion $(\mathrm{BHI})$ broth. An aliquot of $5 \mu \mathrm{l}$ was spotted on $\mathrm{BHI}$ agar plate supplemented with $\mathrm{CR}\left(50 \mu \mathrm{g} \mathrm{ml}^{-1}\right)$ and Coomassie brilliant blue $\left(1 \mathrm{\mu g} \mathrm{ml}^{-1}\right)$ (Zhou et al., 2013; Ching et al., 2019). The plates were incubated at $37^{\circ} \mathrm{C}$ for $48 \mathrm{~h}$.

\section{Autoaggregation assay}

Autoaggregation assays were performed as described elsewhere (Sherlock et al., 2004; Charbonneau et al., 2006) with slight modifications. Bacteria were cultured overnight as 
described above. Overnight cultures were washed twice in PBS, then adjusted to an $\mathrm{OD}_{600}$ of 2.0 in $2 \mathrm{ml}$ of LB in a 17- $x$ $100 \mathrm{~mm}$ culture tube. Cultures were then vortexed for $10 \mathrm{~s}$ and incubated at $37^{\circ} \mathrm{C}$ for $4 \mathrm{~h}$. A $200 \mu$ sample was then taken about $0.5 \mathrm{~cm}$ below the surface and the $\mathrm{OD}_{600}$ was measured at time 0 and $4 \mathrm{~h}$ post-incubation. Percent autoaggregation was calculated by dividing the $\mathrm{OD}_{600}$ value of the aggregated cells by the $O D_{600}$ value of a replicate tube that was vortexed prior to measuring the $\mathrm{OD}_{600}$.

\section{Twitching motility}

The twitching motility assay was performed as described previously (Clemmer et al., 2011; Harding et al., 2013; Biswas et al., 2019), with slight modifications. Briefly, bacteria were cultured overnight in LB broth at $37^{\circ} \mathrm{C}$. Bacterial cultures were resuspended 1:100 in fresh medium, grown to early stationary phase and then adjusted to an $\mathrm{OD}_{600}$ of 2.0. A pipette tip was dipped into the adjusted cultures and used to stab the twitching motility plates to the bottom. Plates were incubated at $37^{\circ} \mathrm{C}$ for $18 \mathrm{~h}$. The motility plates consist of $1 \%$ EIKEN agar and were poured the day of the experiment.

\section{Growth on MacConkey plate}

Bacteria were cultured overnight in LB broth at $37^{\circ} \mathrm{C}$. Bacterial cultures were resuspended 1:100 in fresh medium and grown to mid-log growth phase $\left(\mathrm{OD}_{600}\right.$ of 0.6$)$. Bacteria

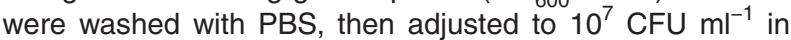
PBS. Serial dilutions were spot plated on MacConkey and incubated for $18 \mathrm{~h}$ at $37^{\circ} \mathrm{C}$. The growth was macroscopically monitored.

\section{Hydrolysis of the chromogenic substrate $X P$}

Bacteria were cultured as described in the CR binding assay section. Five $\mu \mathrm{l}$ of the adjusted cultures were spotted on LB agar supplemented with $100 \mathrm{\mu g} \mathrm{ml}^{-1}$ of XP. The plates were incubated at $37^{\circ} \mathrm{C}$ for $48 \mathrm{~h}$.

\section{Resistance to polymyxin B}

Bacteria were cultured as described in the resistance to normal human serum section, where $10^{7} \mathrm{CFU} \mathrm{\textrm {ml } ^ { - 1 }}$ were incubated with either polymyxin $\mathrm{B}\left(1 \mu \mathrm{g} \mathrm{ml}^{-1}\right)$ or plain LB broth for $60 \mathrm{~min}$. The number of bacteria that survived the treatment was determined by CFU enumeration on LB agar at 15-, 30and 60 min post-incubation.

\section{LOS extraction, electrophoresis and visualization}

Lipooligosaccharide was extracted using the hot aqueousphenol extraction as described by Davis and Goldberg (Davis and Goldberg, 2012), with the exception that LOS was extracted from mid-log phase cultures as described above. Twelve $\mu \mathrm{l}$ of each LOS extraction were loaded on Tricine SDS-PAGE (16\%) for analysis (Lesse et al., 1990). Gels were stained using Pro-Q Emerald 300 LPS gel stain (Invitrogen). Gels were imaged with Bio-Rad and bands were quantified by densitometry using Image Lab version 6.0.1 (Bio-Rad).

\section{Lipid $A$ isolation and analysis by mass spectrometry}

Lipid A was isolated from bacterial cell pellets using an ammonium hydroxide-isobutyric acid-based extraction procedure as previously described (El Hamidi et al., 2005). Briefly, bacterial liquid culture was pelleted and then resuspended in $400 \mu \mathrm{l}$ of $70 \%$ isobutyric acid and $1 \mathrm{M}$ ammonium hydroxide (5:3 vol/vol). Samples were incubated for $1 \mathrm{~h}$ at $100^{\circ} \mathrm{C}$, cooled on ice, and centrifuged at $2,000 \times \mathrm{g}$ for $15 \mathrm{~min}$. Supernatants were transferred to a fresh tube with endotoxin-free water (1:1 vol/vol). Samples were snap-frozen on dry ice and lyophilized overnight. The dried material was washed twice with $1 \mathrm{ml}$ methanol. Lipid A was extracted using $100 \mu \mathrm{l}$ of a chloroform, methanol and water mixture (3:1:0.25 vol/vol/ vol). One $\mu$ l of the lipid A concentrate was spotted on a stainless steel matrix-assisted laser desorption ionization-time of flight (MALDI-TOF) plate followed by $1 \mu \mathrm{l}$ of $10 \mathrm{mg} \mathrm{ml}^{-1}$ norharmane matrix (in chloroform-methanol (2:1 vol/vol)) (Sigma-Aldrich, St. Louis, MO). All samples were analyzed in the negative-ion mode with reflectron mode on a Bruker Microflex mass spectrometer (Bruker Daltonics, Billerica, MA). Mass calibration was achieved using an electrospray tuning mix (Agilent, Palo Alto, CA). All spectral data were analyzed with Bruker Daltonics FlexAnalysis software. The mass spectra were used to evaluate the lipid A structures present based on their predicted structures and molecular weights. Diversity of lipid A structure within a single bacterial membrane is well-described (Dixon and Darveau, 2005; Shaffer et al., 2007).

\section{Bacterial adhesion to hydrocarbons (B.A.T.H)}

The B.A.T.H. assay was performed as described previously (Rosenberg et al., 1980; Goldberg et al., 1990). Briefly, bacteria were cultured to mid-log phase as described above, washed twice in PUM buffer $\left(97.3 \mathrm{mM} \mathrm{K}_{2} \mathrm{HPO}_{4} \cdot 3 \mathrm{H}_{2} \mathrm{O}\right.$, $53.3 \mathrm{mM} \mathrm{KH}_{2} \mathrm{PO}_{4}, 30 \mathrm{mM}$ urea, $1.7 \mathrm{mM} \mathrm{MgSO} \mathrm{M}_{4} \cdot 7 \mathrm{H}_{2} \mathrm{O}$, $\mathrm{pH} 7.1$ ) and resuspended to $10^{8} \mathrm{CFU} \mathrm{\textrm {ml } ^ { - 1 }}$ in PUM buffer. $10^{7} \mathrm{CFU}$ were added to $25 \%$ hydrocarbons (hexadecane or $n$-octane) in PUM buffer, vortex for $2 \mathrm{~min}$ and sit for $15 \mathrm{~min}$ at room temperature. An aliquot from the aqueous phase (lower) was taken, serially diluted and plated on LB agar for CFU enumeration. An increase in cell envelope hydrophobicity is represented by a lower number of CFUs recovered from the aqueous phase.

\section{Antibiotic resistance}

Antibiotic resistance was determined by two approaches. First, minimal inhibitory concentrations (MICs) were determined using E-strips (Biomerieux and Liofilchem). Briefly, bacteria were cultured according to the manufacturer's recommendation and spread on Mueller-Hinton agar plates before a sterile E-test strip was placed in the middle of the plate. Plates were incubated for $18 \mathrm{~h}$ at $37^{\circ} \mathrm{C}$ and the MICs were determined at the concentration where the bacterial growth was inhibited. Second, percent survival to a specific concentration 
of antibiotic was quantified. Bacteria were cultured overnight in LB broth at $37^{\circ} \mathrm{C}$. Bacterial cultures were resuspended $1: 100$ in fresh medium and grown to mid-log growth phase $\left(O D_{600}=0.6\right)$. Cultures were then washed with PBS and adjusted to $10^{7} \mathrm{CFU} \mathrm{ml}{ }^{-1}$ in PBS. Serial dilutions were both plated on LB agar and LB agar supplemented with antibiotic (cefotaxime: $50 \mathrm{\mu g} \mathrm{ml}^{-1}$; carbenicillin: $100 \mathrm{\mu g} \mathrm{ml}^{-1}$ and tetracycline: $20 \mathrm{\mu g} \mathrm{ml}^{-1}$ ). The percent survival was calculated by dividing the number of CFU recovered from the antibiotic plates on the number of CFU enumerated from the LB agar.

\section{Quantitative RT-PCR}

Strains were cultured as described above and RNA was extracted using TRIzol reagent (Thermo Fisher Scientific) according to the manufacturer's recommendations. RNA samples were submitted to a rigorous DNase treatment using Turbo DNA-free (Ambion) to remove any DNA contamination. The iScript cDNA synthesis kit and the SsoFast Evagreen Supermix kit (Bio-Rad) were used for qRT-PCR analysis according to the manufacturer's instructions. The gyrB gene was used as a housekeeping control (Anderson et al., 2017; Crépin et al., 2018). Gene expression was calculated using the $2^{-\Delta \Delta C T}$ method (Livak and Schmittgen, 2001). Genes with a fold-change above or below the defined threshold of two, were considered as differentially expressed. Primers used for qRT-PCR analysis are listed in Table S4.

\section{Statistical analyses}

All data were analyzed by using the GraphPad Prism 8 software program. A Mann-Whitney test was used to determine statistical significance for mono-infection experiments. All other statistical analyses were determined by the Student's $t$-test and either one- or two-way analysis of variance (ANOVA) with Tukey's multiple comparison test.

\section{Acknowledgements}

We would like to thank Sara N. Smith for her assistance during the animal experiments. We are also grateful to Philip N. Rather (Emory University) to kindly share the AB5075 $\triangle$ wZC strain, and Michele S. Swanson (University of Michigan) for sharing her Zeiss Axioplan 2 epifluorescence microscope. This work was supported by the Natural Sciences and Engineering Research Council of Canada PostDoctoral Fellowship (S.C.), the Public Health Service Grant Al107184 from the National Institutes of Health, and the University of Michigan Medical School Host Microbiome Initiative (H.L.T.M.). The funders had no role in the study design, data collection and analysis, decision to publish, or preparation of the manuscript.

\section{Conflict of interest}

The authors declare that they have no conflicts of interest with the contents of this article.

\section{Data availability statement}

The data that support the findings of this study are included in the paper or available from the corresponding authors upon reasonable request.

\section{Author contributions}

S.C., E.N.O, R.K.E. and H.L.T.M. designed the experiments. S.C., E.N.O. and C.E.C. performed the experiments. S.C., E.N.O., R.K.E., and H.L.T.M. analyzed the data. H.L.T.M. and R.K.E. contributed funding and resources. S.C. and H.L.T.M. wrote the manuscript. All authors reviewed, edited and approved the manuscript.

\section{References}

Anderson, M.T., Mitchell, L.A. and Mobley, H.L.T. (2017) Cysteine biosynthesis controls Serratia marcescens phospholipase activity. Journal of Bacteriology, 199(16), e00285-19.

Bartholomew, T.L., Kidd, T.J., Sa Pessoa, J., Conde Alvarez, R. and Bengoechea, J.A. (2019) 2-hydroxylation of Acinetobacter baumannii lipid A contributes to virulence. Infection and Immunity, 87(4), e00066-19.

Belanger, M., Burrows, L.L. and Lam, J.S. (1999) Functional analysis of genes responsible for the synthesis of the B-band $\mathrm{O}$ antigen of Pseudomonas aeruginosa serotype O6 lipopolysaccharide. Microbiology, 145, 3505-3521.

Bishop, R.E. (2005) The lipid A palmitoyltransferase PagP: molecular mechanisms and role in bacterial pathogenesis. Molecular Microbiology, 57, 900-912.

Biswas, I., Machen, A. and Mettlach, J. (2019) In vitro motility assays for Acinetobacter species. Methods in Molecular Biology, 1946, 177-187.

Boll, J.M., Tucker, A.T., Klein, D.R., Beltran, A.M., Brodbelt, J.S., Davies, B.W., et al. (2015) Reinforcing lipid A acylation on the cell surface of Acinetobacter baumannii promotes cationic antimicrobial peptide resistance and desiccation survival. MBio, 6, e00478-e00415.

Castric, P., Cassels, F.J. and Carlson, R.W. (2001) Structural characterization of the Pseudomonas aeruginosa 1244 pilin glycan. Journal of Biological Chemistry, 276, 26479-26485.

Charbonneau, M.E., Berthiaume, F. and Mourez, M. (2006) Proteolytic processing is not essential for multiple functions of the Escherichia coli autotransporter adhesin involved in diffuse adherence (AIDA-I). Journal of Bacteriology, 188, 8504-8512.

Chin, C.Y., Tipton, K.A., Farokhyfar, M., Burd, E.M., Weiss, D.S. and Rather, P.N. (2018) A high-frequency phenotypic switch links bacterial virulence and environmental survival in Acinetobacter baumannii. Nature Microbiology, 3, 563-569.

Ching, C., Yang, B., Onwubueke, C., Lazinski, D., Camilli, A. and Godoy, V.G. (2019) Lon protease has multifaceted biological functions in Acinetobacter baumannii. Journal of Bacteriology, 201(2), e00536-18. 
Choi, A.H., Slamti, L., Avci, F.Y., Pier, G.B. and Maira-Litran, T. (2009) The pgaABCD locus of Acinetobacter baumannii encodes the production of poly-beta-1-6-N-acetylglucosamine, which is critical for biofilm formation. Journal of Bacteriology, 191, 5953-5963.

Clemmer, K.M., Bonomo, R.A. and Rather, P.N. (2011) Genetic analysis of surface motility in Acinetobacter baumannii. Microbiology, 157, 2534-2544.

Conlan, J.W. and North, R.J. (1994) Neutrophils are essential for early anti-Listeria defense in the liver, but not in the spleen or peritoneal cavity, as revealed by a granulocyte-depleting monoclonal antibody. Journal of Experimental Medicine, 179, 259-268.

Crépin, S., Ottosen, E.N., Peters, K., Smith, S.N., Himpsl, S.D., Vollmer, W., et al. (2018) The lytic transglycosylase MltB connects membrane homeostasis and in vivo fitness of Acinetobacter baumannii. Molecular Microbiology, 109(6), 745-762.

Davis, M.R., Jr. and Goldberg, J.B. (2012) Purification and visualization of lipopolysaccharide from Gram-negative bacteria by hot aqueous-phenol extraction. Journal of Visualized Experiments, 63, 3916.

De Silva, P.M., Chong, P., Fernando, D.M., Westmacott, G. and Kumar, A. (2018) Effect of incubation temperature on antibiotic resistance and virulence factors of Acinetobacter baumannii ATCC 17978. Antimicrobial Agents and Chemotherapy, 62(1), e01514-17.

Diderichsen, B. (1980) flu, a metastable gene controlling surface properties of Escherichia coli. Journal of Bacteriology, 141, 858-867.

Dijkshoorn, L., Nemec, A. and Seifert, H. (2007) An increasing threat in hospitals: multidrug-resistant Acinetobacter baumannii. Nature Reviews Microbiology, 5, 939-951.

Dixon, D.R. and Darveau, R.P. (2005) Lipopolysaccharide heterogeneity: innate host responses to bacterial modification of lipid a structure. Journal of Dental Research, 84, 584-595.

van Faassen, H., KuoLee, R., Harris, G., Zhao, X., Conlan, J.W. and Chen, W. (2007) Neutrophils play an important role in host resistance to respiratory infection with Acinetobacter baumannii in mice. Infection and Immunity, 75, 5597-5608.

Fagon, J.Y., Chastre, J., Domart, Y., Trouillet, J.L. and Gibert, C. (1996) Mortality due to ventilator-associated pneumonia or colonization with Pseudomonas or Acinetobacter species: assessment by quantitative culture of samples obtained by a protected specimen brush. Clinical Infectious Diseases, 23, 538-542.

Freeman, D.J., Falkiner, F.R. and Keane, C.T. (1989) New method for detecting slime production by coagulase negative staphylococci. Journal of Clinical Pathology, 42, 872-874.

Garnacho, J., Sole-Violan, J., Sa-Borges, M., Diaz, E. and Rello, J. (2003) Clinical impact of pneumonia caused by Acinetobacter baumannii in intubated patients: a matched cohort study. Critical Care Medicine, 31, 24782482.

Gebhardt, M.J., Gallagher, L.A., Jacobson, R.K., Usacheva, E.A., Peterson, L.R., Zurawski, D.V., et al. (2015) Joint transcriptional control of virulence and resistance to antibiotic and environmental stress in Acinetobacter baumannii. MBio, 6, e01660-e01615.
Geisinger, E. and Isberg, R.R. (2015) Antibiotic modulation of capsular exopolysaccharide and virulence in Acinetobacter baumannii. PLoS Pathogens, 11, e1004691.

Geisinger, E., Huo, W., Hernandez-Bird, J. and Isberg, R.R. (2019) Acinetobacter baumannii: envelope determinants that control drug resistance, virulence, and surface variability. Annual Review of Microbiology, 73, 020518-115714.

Gibson, D.G., Young, L., Chuang, R.-Y., Venter, J.C., Hutchison, C.A. and Smith, H.O. (2009) Enzymatic assembly of DNA molecules up to several hundred kilobases. Nature Methods, 6, 343-345.

Goldberg, S., Doyle, R.J. and Rosenberg, M. (1990) Mechanism of enhancement of microbial cell hydrophobicity by cationic polymers. Journal of Bacteriology, 172, 5650-5654.

El Hamidi, A., Tirsoaga, A., Novikov, A., Hussein, A. and Caroff, M. (2005) Microextraction of bacterial lipid A: easy and rapid method for mass spectrometric characterization. Journal of Lipid Research, 46, 1773-1778.

Hancock, R.E. (1984) Alterations in outer membrane permeability. Annual Review of Microbiology, 38, 237-264.

Harding, C.M., Tracy, E.N., Carruthers, M.D., Rather, P.N., Actis, L.A. and Munson, R.S., Jr. (2013) Acinetobacter baumannii strain M2 produces type IV pili which play a role in natural transformation and twitching motility but not surface-associated motility. MBio, 4(4), e00360-13.

Harding, C.M., Hennon, S.W. and Feldman, M.F. (2018) Uncovering the mechanisms of Acinetobacter baumannii virulence. Nature Reviews Microbiology, 16, 91-102.

Haseley, S.R. and Wilkinson, S.G. (1996) Structure of the O-specific polysaccharide of Acinetobacter baumannii O5 containing 2-acetamido-2-deoxy-D-galacturonic acid. European Journal of Biochemistry, 237, 229-233.

Hashimoto, Y., Li, N., Yokoyama, H. and Ezaki, T. (1993) Complete nucleotide sequence and molecular characterization of ViaB region encoding $\mathrm{Vi}$ antigen in Salmonella typhi. Journal of Bacteriology, 175, 4456-4465.

Iwashkiw, J.A., Seper, A., Weber, B.S., Scott, N.E., Vinogradov, E., Stratilo, C., et al. (2012) Identification of a general O-linked protein glycosylation system in Acinetobacter baumannii and its role in virulence and biofilm formation. PLoS Pathogens, 8, e1002758.

Johnson, E.M., Krauskopf, B. and Baron, L.S. (1965) Genetic mapping of $\mathrm{Vi}$ and somatic antigenic determinants in Salmonella. Journal of Bacteriology, 90, 302-308.

Kamischke, C., Fan, J., Bergeron, J., Kulasekara, H.D., Dalebroux, Z.D., Burrell, A., et al. (2019) The Acinetobacter baumannii Mla system and glycerophospholipid transport to the outer membrane. Elife, 8, e40171.

Karalewitz, A.P. and Miller, S.I. (2018) Multidrug-resistant Acinetobacter baumannii chloramphenicol resistance requires an inner membrane permease. Antimicrobial Agents and Chemotherapy, 62(8), e00513-18.

Kenyon, J.J. and Hall, R.M. (2013) Variation in the complex carbohydrate biosynthesis loci of Acinetobacter baumannii genomes. PLoS One, 8(4), e62160.

Kenyon, J.J., Shneider, M.M., Senchenkova, S.N., Shashkov, A.S., Siniagina, M.N., Malanin, S.Y., et al. (2016) K19 capsular polysaccharide of Acinetobacter baumannii is produced via a Wzy polymerase encoded in a small genomic island rather than the KL19 capsule gene cluster. Microbiology, 162, 1479-1489. 
Klein, D.R., Powers, M., Trent, M.S. and Brodbelt, J.S. (2019) Top-down characterization of lipooligosaccharides from antibiotic-resistant bacteria. Analytical Chemistry, 91(15), 9608-9615.

Knirel, Y.A. (1990) Polysaccharide antigens of Pseudomonas aeruginosa. Critical Reviews in Microbiology, 17, 273-304.

Lam, J.S., Taylor, V.L., Islam, S.T., Hao, Y. and Kocincova, D. (2011) Genetic and functional diversity of Pseudomonas aeruginosa lipopolysaccharide. Frontiers in Microbiology, 2, 118.

Lamers, R.P., Nguyen, U.T., Nguyen, Y., Buensuceso, R.N. and Burrows, L.L. (2015) Loss of membrane-bound lytic transglycosylases increases outer membrane permeability and beta-lactam sensitivity in Pseudomonas aeruginosa. Microbiologyopen, 4, 879-895.

Lane, M.C., Simms, A.N. and Mobley, H.L.T. (2007) Complex interplay between type 1 fimbrial expression and flagellum-mediated motility of uropathogenic Escherichia coli. Journal of Bacteriology, 189, 5523-5533.

Lawe-Davies, O. and Bennett, S. (2017) WHO publishes list of bacteria for which new antibiotics are urgently needed. Available at: https://www.who.int/en/news-room/detail/2702-2017-who-publishes-list-of-bacteria-for-which-newan tibiotics-are-urgently-needed. Accessed 25 October 2019.

Lees-Miller, R.G., Iwashkiw, J.A., Scott, N.E., Seper, A., Vinogradov, E., Schild, S., et al. (2013) A common pathway for O-linked protein-glycosylation and synthesis of capsule in Acinetobacter baumannii. Molecular Microbiology, 89, 816-830.

Lesse, A.J., Campagnari, A.A., Bittner, W.E. and Apicella, M.A. (1990) Increased resolution of lipopolysaccharides and lipooligosaccharides utilizing tricine-sodium dodecyl sulfate-polyacrylamide gel electrophoresis. Journal of Immunological Methods, 126, 109-117.

Leung, L.M., McElheny, C.L., Gardner, F.M., Chandler, C.E., Bowler, S.L., Mettus, R.T., et al. (2019) A prospective study of Acinetobacter baumannii complex isolates and colistin susceptibility monitoring by mass spectrometry of microbial membrane glycolipids. Journal of Clinical Microbiology, 57(3), e01100-18.

Livak, K.J. and Schmittgen, T.D. (2001) Analysis of relative gene expression data using real-time quantitative PCR and the 2(-Delta Delta C(T)) method. Methods, 25, 402-408.

Maneval, W.E. (1941) Staining bacteria and yeasts with acid dyes. Stain Technology, 16, 13-19.

Mattick, J.S. (2002) Type IV pili and twitching motility. Annual Review of Microbiology, 56, 289-314.

Mitchell, A.M. and Silhavy, T.J. (2019) Envelope stress responses: balancing damage repair and toxicity. Nature Reviews Microbiology, 17(7), 417-428.

Munoz-Price, L.S. and Weinstein, R.A. (2008) Current concepts: Acinetobacter infection. New England Journal of Medicine, 358, 1271-1281.

Murray, G.L., Tsyganov, K., Kostoulias, X.P., Bulach, D.M., Powell, D., Creek, D.J., et al. (2017) Global gene expression profile of Acinetobacter baumannii during bacteremia. Journal of Infectious Diseases, 215, S52-S57.

Oyler, B.L., Khan, M.M., Smith, D.F., Harberts, E.M., Kilgour, D.P.A., Ernst, R.K., et al. (2018) Top down tandem mass spectrometric analysis of a chemically modified rough-type lipopolysaccharide vaccine candidate. Journal of the American Society for Mass Spectrometry, 29, 1221-1229.

Pelletier, M.R., Casella, L.G., Jones, J.W., Adams, M.D., Zurawski, D.V., Hazlett, K.R., et al. (2013) Unique structural modifications are present in the lipopolysaccharide from colistin-resistant strains of Acinetobacter baumannii. Antimicrobial Agents and Chemotherapy, 57, 4831-4840.

Powers, M.J. and Trent, M.S. (2018) Expanding the paradigm for the outer membrane: Acinetobacter baumannii in the absence of endotoxin. Molecular Microbiology, 107, 47-56.

Raetz, C.R. and Whitfield, C. (2002) Lipopolysaccharide endotoxins. Annual Review of Biochemistry, 71, 635-700.

Romling, U., Sierralta, W.D., Eriksson, K. and Normark, S. (1998) Multicellular and aggregative behaviour of Salmonella typhimurium strains is controlled by mutations in the agfD promoter. Molecular Microbiology, 28, 249-264.

Rosenberg, M., Gutnick, D. and Rosenberg, E. (1980) Adherence of bacteria to hydrocarbons: a simple method for measuring cell-surface hydrophobicity. FEMS Microbiology Letters, 9, 29-33.

Runci, F., Gentile, V., Frangipani, E., Rampioni, G., Leoni, L., Lucidi, M., et al. (2019) Contribution of active iron uptake to Acinetobacter baumannii pathogenicity. Infection and Immunity, 87(4), e00755-18.

Russo, T.A., Beanan, J.M., Olson, R., MacDonald, U., Cox, A.D., St Michael, F., et al. (2013) The K1 capsular polysaccharide from Acinetobacter baumannii Is a potential therapeutic target via passive immunization. Infection and Immunity, 81, 915-922.

Schembri, M.A., Kjaergaard, K. and Klemm, P. (2003) Global gene expression in Escherichia coli biofilms. Molecular Microbiology, 48, 253-267.

Schembri, M.A., Dalsgaard, D. and Klemm, P. (2004) Capsule shields the function of short bacterial adhesins. Journal of Bacteriology, 186, 1249-1257.

Seifert, H., Strate, A. and Pulverer, G. (1995) Nosocomial bacteremia due to Acinetobacter-baumannii. Clinical features, epidemiology, and predictors of mortality. Medicine, 74, 340-349.

Shaffer, S.A., Harvey, M.D., Goodlett, D.R. and Ernst, R.K. (2007) Structural heterogeneity and environmentally regulated remodeling of Francisella tularensis subspecies novicida lipid A characterized by tandem mass spectrometry. Journal of the American Society for Mass Spectrometry, 18, 1080-1092.

Shashkov, A.S., Liu, B., Kenyon, J.J., Popova, A.V., Shneider, M.M., Senchenkova, S.N., et al. (2017) Structures of the K35 and K15 capsular polysaccharides of Acinetobacter baumannii LUH5535 and LUH5554 containing amino and diamino uronic acids. Carbohydrate Research, 448, 28-34.

Shashkov, A.S., Kenyon, J.J., Arbatsky, N.P., Shneider, M.M., Popova, A.V., Knirel, Y.A., et al. (2018) Genetics of biosynthesis and structure of the K53 capsular polysaccharide of Acinetobacter baumannii D23 made up of a disaccharide $\mathrm{K}$ unit. Microbiology, 164, 1289-1292.

Sherlock, O., Schembri, M.A., Reisner, A. and Klemm, P. (2004) Novel roles for the AIDA adhesin from diarrheagenic Escherichia coli: cell aggregation and biofilm formation. Journal of Bacteriology, 186, 8058-8065. 
Simpson, B.W. and Trent, M.S. (2019) Pushing the envelope: LPS modifications and their consequences. Nature Reviews Microbiology, 7, 403-416.

Singh, J.K., Adams, F.G. and Brown, M.H. (2019) Diversity and function of capsular polysaccharide in Acinetobacter baumannii. Frontiers in Microbiology, 9, 3301.

Smith, S.N., Hagan, E.C., Lane, M.C. and Mobley, H.L. (2010) Dissemination and systemic colonization of uropathogenic Escherichia coli in a murine model of bacteremia. MBio, 1(5), e00262-10.

Spellberg, B. and Rex, J.H. (2013) The value of single-pathogen antibacterial agents. Nature Reviews Drug Discovery, 12, 963-964.

Subashchandrabose, S., Smith, S., DeOrnellas, V., Crepin, S., Kole, M., Zahdeh, C., et al. (2016) Acinetobacter baumannii genes required for bacterial survival during bloodstream infection. Msphere, 1(1), e00013-15.

Tipton, K.A. and Rather, P.N. (2019) Extraction and visualization of capsular polysaccharide from Acinetobacter baumannii. Methods in Molecular Biology, 1946, 227-231.

Tipton, K.A., Chin, C.-Y., Farokhyfar, M., Weiss, D.S. and Rather, P.N. (2018) Role of capsule in resistance to disinfectants, host antimicrobials, and desiccation in Acinetobacter baumannii. Antimicrobial Agents and Chemotherapy, 62(12), e01188-18.

Todor, H., Dulmage, K., Gillum, N., Bain, J.R., Muehlbauer, M.J. and Schmid, A.K. (2014) A transcription factor links growth rate and metabolism in the hypersaline adapted archaeon Halobacterium salinarum. Molecular Microbiology, 93, 1172-1182.

Tonner, P.D., Pittman, A.M., Gulli, J.G., Sharma, K. and Schmid, A.K. (2015) A regulatory hierarchy controls the dynamic transcriptional response to extreme oxidative stress in archaea. PLoS Genetics, 11, e1004912.

Ulett, G.C., Webb, R.I. and Schembri, M.A. (2006) Antigen-43mediated autoaggregation impairs motility in Escherichia coli. Microbiology, 152, 2101-2110.

Valle, J., Mabbett, A.N., Ulett, G.C., Toledo-Arana, A., Wecker, K., Totsika, M., et al. (2008) UpaG, a new member of the trimeric autotransporter family of adhesins in uropathogenic Escherichia coli. Journal of Bacteriology, 190, 4147-4161.

Vinogradov, E.V., Brade, L., Brade, H. and Holst, O. (2003) Structural and serological characterisation of the O-antigenic polysaccharide of the lipopolysaccharide from Acinetobacter baumannii strain 24. Carbohydrate Research, 338, 2751-2756.

Waack, U., Warnock, M., Yee, A., Huttinger, Z., Smith, S., Kumar, A., et al. (2018) CpaA is a glycan-specific adamalysin-like protease secreted by Acinetobacter baumannii that inactivates coagulation factor XII. MBio, 9(6), e01606-18.

Wang, N., Ozer, E.A., Mandel, M.J. and Hauser, A.R. (2014) Genome-wide identification of Acinetobacter baumannii genes necessary for persistence in the lung. MBio, 5, e01163-e01114.

Waxin, H., Virlogeux, I., Kolyva, S. and Popoff, M.Y. (1993) Identification of six open reading frames in the Salmonella enterica subsp. enterica ser. Typhi viaB locus involved in $\mathrm{Vi}$ antigen production. Research in Microbiology, 144, 363-371.

Whitfield, C. (2006) Biosynthesis and assembly of capsular polysaccharides in Escherichia coli. Annual Review of Biochemistry, 75, 39-68.

Whitfield, C. and Paiment, A. (2003) Biosynthesis and assembly of Group 1 capsular polysaccharides in Escherichia coli and related extracellular polysaccharides in other bacteria. Carbohydrate Research, 338, 2491-2502.

Willyard, C. (2017) The drug-resistant bacteria that pose the greatest health threats. Nature, 543, 15.

Wisplinghoff, H., Bischoff, T., Tallent, S.M., Seifert, H., Wenzel, R.P. and Edmond, M.B. (2004) Nosocomial bloodstream infections in US hospitals: analysis of 24,179 cases from a prospective nationwide surveillance study. Clinical Infectious Diseases, 39, 309-317.

Wong, D., Nielsen, T.B., Bonomo, R.A., Pantapalangkoor, P., Luna, B. and Spellberg, B. (2017) Clinical and pathophysiological overview of Acinetobacter infections: a century of challenges. Clinical Microbiology Reviews, 30, 409-447.

Xu, Q., Chen, T., Yan, B., Zhang, L., Pi, B., Yang, Y., et al. (2019) Dual role of gnaA in antibiotic resistance and virulence in Acinetobacter baumannii. Antimicrobial Agents and Chemotherapy, 63(10), e00694-19.

Zhang, H., Zhou, Y., Bao, H. and Liu, H.W. (2006) Vi antigen biosynthesis in Salmonella typhi: characterization of UDP$\mathrm{N}$-acetylglucosamine C-6 dehydrogenase (TviB) and UDP$\mathrm{N}$-acetylglucosaminuronic acid C-4 epimerase (TviC). Biochemistry, 45, 8163-8173.

Zhou, Y., Smith, D.R., Hufnagel, D.A. and Chapman, M.R. (2013) Experimental manipulation of the microbial functional amyloid called curli. Methods in Molecular Biology, 966, 53-75.

\section{Supporting Information}

Additional supporting information may be found online in the Supporting Information section at the end of the article. 